Erschienen in: Deppermann, Arnulf/Linke, Angelika (Hrsg.): Sprache intermedial. Stimme und Schrift, Bild und Ton. - Berlin/New York: de Gruyter, 2010. S. 325-358. (Jahrbuch des Instituts für Deutsche Sprache 2009), https://doi.org/10.1515/9783110223613.325

Martin Steinseifer

\title{
Prägnanzen
}

\section{Bilder und ihre Effekte in der pragmatischen Linguistik}

\begin{abstract}
Der Beitrag nimmt die verbreitete Annahme einer besonderen Überzeugungskraft von Bildern zum Anlass, nach dem Ort einer solchen medienspezifischen Wirkungsweise im Rahmen einer pragmatischen Linguistik zu fragen. In exemplarischen Analysen wird gezeigt, wie Fotografien in journalistischen Printmedien eingesetzt werden, um Bedeutungen, die ihnen durch die umstehenden Texte ,zugeschrieben' und so stabilisiert werden, ein besonderes Maß an Plausibilität zu verleihen. Dieser inter-mediale Evidenzeffekt wird als Prägnanz bezeichnet. Zu seiner theoretischen Begründung wird der Peircesche Begriff der Ikonizität mit dem Konzept der ,ikonischen Differenz ${ }^{6}$ aus der phänomenologischen Bildtheorie Gottfried Boehms verbunden. Denn beide stellen heraus, dass die wahrnehmbaren Eigenschaften der Zeichenform ein notwendiges Komplement zu symbolischen Schematisierungen im Prozess der Sinnkonstitution bilden. Diese Verschränkung zwischen Ikonizität und Symbolizität prägt sowohl die Konstitution dessen, was ein gegenständliches Bild in seinen Teilen wiedererkennbar zeigt, als auch dessen, was es als Ganzes - durch die Anordnung von Elementen auf einer begrenzten Fläche - darstellt. Die sichtbare Form dieser Anordnung wird als Grundlage für das besondere Prägnanzpotenzial von Bildern in der Zusammenstellung mit Texten identifiziert. Gestützt auf Beispiele wird zwischen einer schematischen und ikonischen Variante der Prägnanz unterschieden und das methodische Vorgehen reflektiert. Die pragmatische ,Effektivität ${ }^{6}$ der wahrnehmbaren Zeichenform, die in den Varianten der Prägnanz fassbar wird, ist allerdings nicht auf die intermediale Konstellation von Text und Bild beschränkt, weswegen der Beitrag mit dem Ausblick auf eine linguistische Phänomenologie der Textgestalt schließt.
\end{abstract}

\section{Hinführung}

Es gehört zum gesellschaftlichen Common sense, dass Bilder auf eine besondere Weise wirken. Für dieses Wissen steht unter anderem die Rede von der Macht der Bilder. Diese Redeweise fungiert als eine Art Beschwörungsformel, um Bilder entweder besonders attraktiv oder besonders gefährlich erscheinen zu lassen. Mit Blick auf die Formel ist allerdings weniger der beschwörende Gestus von Interesse, sondern vielmehr der Hinweis auf eine besondere mediale Wirkungsweise, durch die sich Bilder auszeichnen zielt er doch auf kommunikative Effekte, die von bestimmten visuell wahrzunehmenden Eigenschaften der Zeichenform ausgehen. Bildliches lenkt in anderer Weise als Sprachliches den Blick auf die Oberfläche. Ein verstärktes ,Augenmerk' auf Qualitäten der Zeichenform ist jedoch auch für eine pragmatische Linguistik notwendig, die sich Wörter wie Medialität, Medienspezifik, multimediale oder multimodale Kommunikation auf die Fahnen schreibt. 
Nachdem es lange Zeit kaum ein linguistisches Interesse an Bildern und ihrem Verhältnis zu sprachlichen Ausdrucksformen oder sprachlichen Handlungen gab - Manfred Muckenhaupts grundlegende Arbeit zu Text und Bild (1986) war im Bereich der germanistischen Linguistik eine Ausnahmeerscheinung - ändert sich diese Situation seit einigen Jahren. Die Gründe dafür dürften ebenso in Veränderungen des (massen-)medialen wie des akademischen Kommunikationsalltags zu suchen sein, die auf neue technische Möglichkeiten der Herstellung und Verbreitung von Bildern sowie deren Verbindung mit Schriftzügen und Texten zu „Sehflächen“ zurückgehen. ${ }^{1}$ Nicht nur das World Wide Web bietet sich seinen Nutzerinnen und Nutzern als eine Vielzahl solcher Sehflächen dar, auch auf Tagungen und in Lehrveranstaltungen ist es inzwischen fast obligatorisch, mündlichen Vorträgen durch begleitende Sehflächen die Form einer multimodalen ,Präsentation' zu geben - relativ unabhängig von der Rolle, die Bilder und ,bildgebende Verfahren' im wissenschaftlichen Erkenntnisprozess spielen. Die linguistische Hinwendung zu Bildern ist daher auch kein Einzelfall. Sie findet im Zusammenhang mit einem disziplinübergreifend wachsenden Interesse an Bildern und Bildlichkeit statt, das seit den 1990er Jahren als pictorial (Mitchell 1994) bzw. iconic turn (Boehm 1994) der Kulturwissenschaften propagiert wurde. Dieses neue Interesse gewinnt im englischsprachigen Raum unter den Bezeichnungen Visual Culture bzw. Visual Studies ${ }^{2}$ institutionelle Gestalt, während es im deutschsprachigen Raum in den kontroversen Bemühungen um eine Bildwissenschaft zu greifen ist, die das etablierte Gegenstandsfeld der Kunstgeschichte überschreitet. ${ }^{3}$

Betrachtet man die linguistischen Ansätze der letzten Jahre in diesem weiteren Rahmen, dann fällt die zentrale Rolle auf, die für sie eine semantische und funktionale Integration von Text und Bild spielt, wobei die kom-

1 „Sehflächen lesen“ ist der Titel eines Themenhefts der Zeitschrift Der Deutschunterricht (2005, Ulrich Schmitz/Ursula Renner), das sich dem Verhältnis und den Verbindungen von Sprache und Bild widmet. Neben diesem geben die beiden Themenhefte der Mitteilungen des Deutschen Germanistenverbandes „Sprache und Bild“ (51 (1+2)/2004, Werner Holly/Almuth Hoppe/Ulrich Schmitz) und der Zeitscbrift für Germanistische Linguistik, „Schrift - Text - Bild“ (2007, Christa Dürscheid), sowie der Band Schmitz/Wenzel (2003) einen Einblick in die aktuelle Diskussion der deutschsprachigen Linguistik. Als wichtige Impulsgeber fungieren die schon genannten Ulrich Schmitz $(2003,2005,2008)$ und Werner Holly $(2005,2009)$, sowie Hartmut Stöckl, insbesondere mit seiner Monographie „Die Sprache im Bild - das Bild in der Sprache“ (2004a).

2 Vgl. für einen Überblick über das keineswegs homogene Feld die Einleitung in Elkins (2003), sowie die Debatte, die im Jorunal of Visual Culture im Anschluss an einen Aufsatz von Mieke Bal (2003) geführt wurde. In den Reaktionen von Norman Bryson, James Elkins, William J.T. Mitchell und anderen wird die Spannbreite von Objekten, Theorien und Methodologien deutlich.

3 Neben den unterschiedlichen Ansätzen aus dem Bereich der Kunstgeschichte von Bredekamp (2003), Belting (2001) und Boehm (2007a) ist hier insbesondere der programmatische philosophische Entwurf einer ,allgemeinen Bildwissenschaft" von Sachs-Hombach (2003) zu nennen. 
munikative Einheit aus bildlichen und sprachlichen Teilen zumeist wiederum als Text verstanden wird - Fix (1996, S. 116) etwa spricht von einem „Supertext“", Stöckl (2004a, S. 97 u.ö.) vom „Gesamttext“". Sie fällt auf, weil in den bildwissenschaftlichen Projekten nicht nur das Zusammenspiel von Bildern und Texten kein derart bevorzugter Untersuchungsgegenstand ist, wie für eine sich dem Visuellen öffnende Sprachwissenschaft, sondern weil die Beschäftigung mit der sichtbaren Form zudem immer wieder zur scharfen Abgrenzung von Bild und Sprache führt, die als Ausdrucksformen mit einer je eigenen medialen Wirkungsweise angesehen werden. Auch wenn diese Abgrenzung in erster Linie darauf zielt, Bilder gegenüber einer sprachzentrierten philosophischen Tradition als eigenständiges Erkenntnismedium zu rehabilitieren, so betrifft sie doch auch die linguistischen Bemühungen um ein semiotisch begründetes Verständnis von Bildern als Texten, die leicht unter den Verdacht der Fortschreibung eines solchen Linguozentrismus geraten. Man muss beide Vorgehensweisen allerdings nicht als sich wechselseitig ausschließende Alternativen begreifen, sondern kann sie auch als unterschiedlich akzentuierte Konsequenzen aus zwei gemeinsamen Grundeinsichten auffassen: Auf der einen Seite besteht Einigkeit darin, dass Bilder eine unersetz- und unverzichtbare Rolle im kommunikativen Haushalt nicht erst unserer spätmodernen Gesellschaft spielen und daher mehr als bisher der Analyse bedürfen. Auf der anderen Seite besteht weitgehende Einigkeit darin, dass Bilder keine der doppelten Artikulation und der propositionalen Struktur sprachlicher Zeichen entsprechende Struktur aufweisen und es daher auch keine der Sprache vergleichbare Grammatik bzw. Syntax des Bildes gibt. ${ }^{4}$

In diesem Beitrag soll daher ein pragmatischer Theorierahmen skizziert werden, in dem sich beide Vorgehensweisen aufeinander beziehen lassen, wobei sich das alltägliche Zusammenspiel von Bildern und Texten gerade als Kehrseite der medialen Eigenständigkeit erweisen wird. Den Ausgangspunkt dafür bildet die pragmatistische Zeichenphilosophie von Charles Sanders Peirce - insbesondere seine bekannte Trichonomie von Ikon, Index und Symbol. Diese Trias ist allerdings nur dann weiterführend, wenn die mit ihr verbundene logische Unterscheidung von Möglichkeiten der Objektrelation im Zeichenprozess nicht, wie es keineswegs nur in der Linguistik verbreitet ist, in erster Linie zur Bildung unterschiedlicher Zeichenklassen verwendet wird, sondern Ikonizität, Indexikalität und Symbolizität

Die Versuche der strukturalistischen Bildsemiotik während der 1970er Jahre, eine kombinatorische Syntax des Bildes zu entwickeln, konnten sich nicht durchsetzen. Vgl. dazu die knappen Bemerkungen bei Nöth (2000, S. 478 ff.) sowie die Kritik bei Sonesson (1993) und Sachs-Hombach (2003, S. 100 ff.), der seiner Arbeit leider dennoch Morris' Trennung in Syntax, Semantik und Pragmatik zu Grunde legt. Die Grammar of Visual Design von Gunter Kress und Theo van Leeuwen (1996) ist gerade keine Fortschreibung dieser strukturalistischen Tradition einer formalen, kompositorischen Bildsyntax, sondern eine Weiterentwicklung der funktional begründeten social semiotics Michael A.K. Hallidays. 
als drei konstitutive Dimensionen jeder Semiose - dem Prozess der Verbindung eines Repräsentamens (einer materiellen Zeichenform) mit einem Objekt durch einem Interpretanten - verstanden werden. ${ }^{5}$ Dann liefert nämlich die ,Ikonizität den Ansatzpunkt für eine Pbänomenologie der Zeichenform, die eine Analyse der symbolischen Momente bei der Etablierung von Bedeutungen ergänzen kann und so zu einem Verständnis der spezifischen medialen Wirkungsweise von Bildern hinführt. ${ }^{6}$ Die konsequente Verbindung von Ikonizität und Symbolizität eröffnet nämlich die Möglichkeit, wichtige Einsichten auch von solchen Ansätzen der Bildtheorie aus dem Bereich der Kunstgeschichte und Philosophie aufzunehmen, die selbst eine phänomenologische Vorgehensweise als Alternative zur Semiotik propagieren. Das soll am Beispiel der Bildtheorie von Gottfried Boehm erläutert werden.

Zur Verdeutlichung der Argumentation werden Beispiele der Verwendung von Fotografien in journalistischen Printmedien herangezogen. Das ist ein Gegenstand, dem eine bildtheoretisch ausgerichtete Kunstgeschichte bisher kein großes Interesse entgegengebracht hat - wohl weil sie diese Fotografien primär als illustrative Abbildungen ohne großen ästhetischen Eigenwert ansieht. Und in der Tat kann eine semantische Analyse des TextBild-Verhältnisses in vielen Fällen Dopplungen oder Redundanzen ${ }^{7}$ konstatieren. In geradezu plakativer Weise ist das etwa bei einer Titelseite des SPIEGEL aus dem Mai des Jahres 1972 der Fall (Abb. 1). Die Titelzeile lautet „Bomben in der Bundesrepublik“ und sie ist in ein Bild gesetzt, das drei

5 Eine klassifizierende Verwendung, die auf Peirce eigene Bemühungen um eine tragfähige Klassifikation von Zeichenphänomenen zurückgeht, findet sich etwa im Semiotik-Kapitel des Studienbuchs Linguistik (Linke/Nussbaumer/Portmann 2004, S. 19), ihr Status als semiotisches Allgemeingut zeigt sich aber auch darin, dass in Winfried Nöths Handbuch der Semiotik (2000, S. 178 ff.) Symbol, Index und Ikon je ein eigenes Kapitel gewidmet ist. Gerade im Kapitel zum Ikon deutet jedoch auch Nöth an, dass die Verwendung der Termini zu differenzieren ist, wenn man sie in Bezug setzt zu den drei universalen Kategorien, die den Ausgangs- und Bezugspunkt der Peirceschen Zeichenphilosophie bilden. Während nämlich einerseits die möglichen Zeichenklassen logisch aus den Kategorien abgeleitet werden (vgl. etwa Nomenklatur und Unterteilung der triadischen Relationen, 1903 (Peirce 1983, S. 121-138)), ist jede wirkliche Semiose durch ein Zusammenspiel aller Dimensionen gekennzeichnet. Das verdeutlichen immer wieder auch die Analysen von konkreten Zeichenverwendungen, die sich in Peirces Texten jenseits der definitorischen Passagen finden. Vgl. etwa seine Ausführungen zum gemalten Porträt einer Person in den Entwïrfen für ein Logikbuch, MS 425 A, 1902 (Peirce 1986, S. 391), auf die ich weiter unten in Abschnitt 1 zurückkomme.

6 Auf eine Diskussion der indexikalischen Dimension und ihres Verhältnisses zu den beiden anderen Dimensionen der Objektrelation muss im Rahmen dieses Aufsatzes verzichtet werden. Der Anteil, den sie gerade im Fall von Fotografien an der besonderen Überzeugungskraft von Bildern hat, soll damit allerdings keineswegs bestritten werden. Vgl. dazu Steinseifer (im Erscheinen) sowie die Überlegungen zur ,indexikalischen Evidenz“ bei Jäger (2008). Seine auf Peirce gestützte Kritik an der späten fototheoretischen Position von Barthes ([1980] 1985) kann in großen Teilen komplementär zu der hier entwickelten Argumentation gelesen werden.

7 So der semiotische Terminus, der auf Barthes ([1964] 1990, S. 38) zurückgeht. Stöckl (2004a, S. 254) bezeichnet diesen ,unauffälligen Normalfall“" als Parallelisierung. 
solche Bomben zeigt. Das Bild verdoppelt hier also einen Teil der Bedeutung, die auch mit sprachlichen Mitteln zum Ausdruck gebracht wird. Allerdings muss man sich gerade angesichts solcher überdeutlichen Fälle von Verdopplung fragen, warum Bilder denn dann überhaupt verwendet werden, und vermuten, dass der Grund dafür nicht einfach in ihrer Semantik liegen kann. Fällt sie als Grund aus, rücken pragmatische Fragen notwendig in den Mittelpunkt: Ein Grund für die Verwendung von Bildern ist die spezifische Überzeugungskraft, die Bilder dem verleihen, was sie im jeweiligen Zusammenhang darstellen. Für diese Überzeugungskraft soll hier die Bezeichnung Prägnanz vorgeschlagen werden.

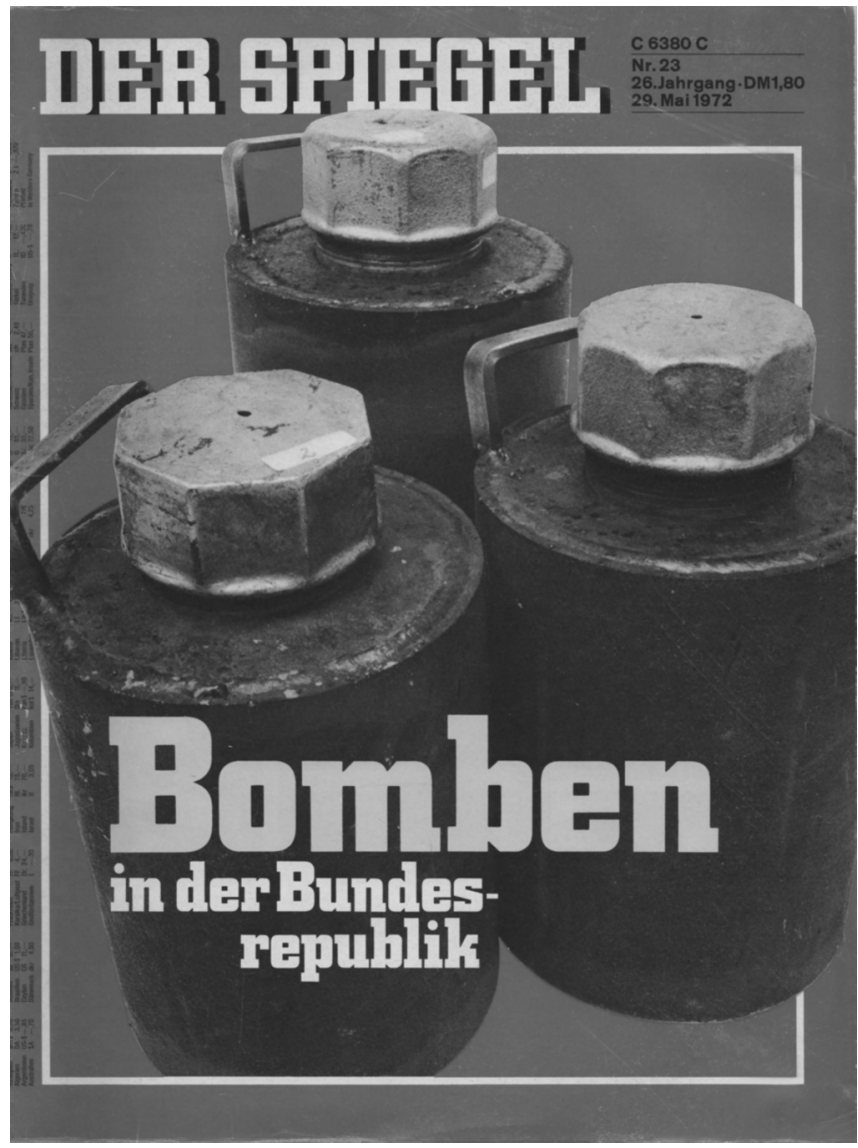

Abb. 1: SPIEGEL 23, 29.5.1972, Titel

Wenn als Beispiele Beiträge aus der Auseinandersetzung mit ,Terrorismus ${ }^{c}$ in der Bundesrepublik von vor 30 Jahren herangezogen werden, hat das allerdings einen weiteren Grund. Im Hinblick auf diesen „linken Ter- 
rorismus ${ }^{\text {“8 }}$ wie auch auf andere brisante und gesellschaftlich folgenreiche Ereigniszusammenhänge, wird nämlich eine zweite Frage wichtig, die sich ebenfalls nur pragmatisch beantworten lässt: Wie kommt es dazu, dass aus der enormen Zahl von publizierten Bildern nur bestimmte wiederverwendet werden, die dann im kollektiven Gedächtnis für ein Ereignis stehen? Zu diesen bis heute bekannten Bildern des ,Terrorismus'-Diskurses gehören die ,Bomben` des SPIEGEL-Titels sicher nicht, wohl aber das folgende Bild vom Ort der Entführung des damaligen Arbeitgeberpräsidenten HannsMartin Schleyer durch ein Kommando der, Roten Armee Fraktion' am 5. September 1977, das kurze Zeit nach dem Attentat in der Illustrierten STERN erschien (Abb. 2). Dafür dass sich dieses Bild als ,Foto-Ikone' (vgl. Brink 1998; Koetzle 2002) diskursgeschichtlich durchgesetzt hat, ist nicht nur die Brutalität der dargestellten Gewaltereignisse und der Stellenwert des sogenannten „Deutschen Herbstes“ im Terrorismus-Zusammenhang der 1970er Jahre verantwortlich. Es wird sich zeigen, dass neben diesen bildexternen auch bestimmte bildliche Eigenschaften entscheidend sind, die für ein besonderes Prägnanzpotenzial sorgen.

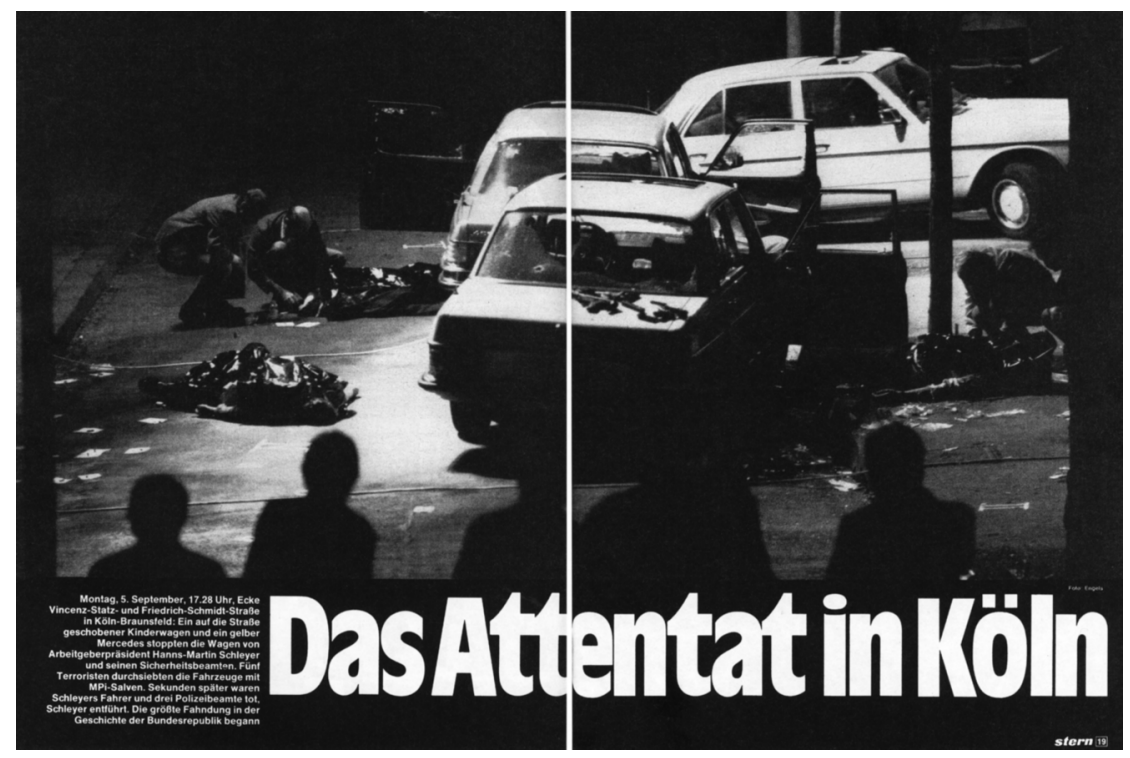

Abb. 2: STERN 38, 8.9.1977, S. 18-19

Fotografien in Printmedien sind aber noch aus einem dritten Grund interessant: Der diskursive Zusammenhang, in dem Bilder etwas darstellen, wird hier nämlich gerade nicht allein durch Bilder konstituiert, sondern

$8 \quad$ So die Kategorisierung im Titel der zweibändigen Geschichte dieses Ereigniszusammenhangs, die am Hamburger Institut für Sozialforschung entstanden ist (Kraushaar 2006). 
ebenso - und in erster Linie - durch schriftliche Texte. Entsprechend muss auch die Prägnanz als inter-mediale Größe verstanden und analysiert werden. Sie ist ein Effekt, der im Zusammenspiel ,zwischen' Textteilen und Bildern entsteht, wenn diese als Teile eines Kommunikationsangebots ${ }^{9}$ verstanden werden, das damit zu einem Beitrag in einem Diskurszusammenhang wird. Erst in einem solchen (thematisch bestimmten) kommunikativen Verwendungszusammenhang kann sich das spezifische Prägnanzpotenzial eines Bildes auswirken. Die Frage nach der spezifischen Wirkungsweise von Bildern ist also gerade nicht durch ihre isolierte Betrachtung zu beantworten, sondern in einer pragmatischen Perspektive rückt sie die wechselseitige Bezogenheit der beiden Ausdrucksformen Sprache und Bild in den Blick. Die Beziehung von Text und Bild lässt sich semiotisch rekonstruieren, sie ist allerdings gerade nicht symmetrisch: Sondern indem Texte bzw. Textteile in Text-Bild-Zusammenstellungen die Darstellungsbedeutung der verwendeten Bilder stabilisieren, profitieren sie umgekehrt von der Überzeugungskraft, die von wahrnehmbaren Eigenschaften der Zeichenform der Bilder ausgehen.

Dieser Zusammenhang soll im Folgenden in drei Schritten entfaltet werden: Zunächst wird die ,ikonische Differenz', die im Zentrum der Bildtheorie Gottfried Boehms steht, auf die ikonische Dimension der Semiose bei Peirce bezogen, um so die Rolle von Eigenschaften der sichtbaren Zeichenform hervortreten zu lassen (1). Dabei werden zugleich die Unterschiede bildlicher Sinnkonstitution zu außerbildlichen Wahrnehmungsprozessen deutlich, so dass in einem zweiten Schritt eine Sinn- bzw. Bedeutungsebene eingeführt wird, die der spezifischen Verschränkung ikonischer und symbolischer Momente bei gegenständlichen Bildern Rechnung trägt und die als Ebene der ,Darstellung' bezeichnet wird (2). Auf dieser Basis können dann in einem dritten Schritt zwei idealtypische Varianten von Prägnanzeffekten in Text-Bild-Zusammenstellungen unterschieden werden (3). Die Frage nach,Qualität ${ }^{`}$ und ,Effektivität ${ }^{`}$ der wahrnehmbaren Zeichenformen ist aber nicht auf die inter-mediale Konstellation von Text und Bild beschränkt. Als Frage nach der medialen, Wirkungsweise' kann sie ebenso an sprachliche Zeichenformen gestellt werden. Der Ausblick auf eine pragmatisch-linguistische Phänomenologie der Textgestalt wird daher den Beitrag beschließen (4).

Kommunikationsangebot ist eine medienneutrale Bezeichnung für das wahrnehmbare Ganze, das als Träger von Text und Bild fungiert. Schmidt ([1980] 1991, S. 61 f.) bestimmt solche „phyischen Wahrnehmungsangebote“, die als Mittel kommunikativer Handlungen dienen, ebenfalls medienneutral als Kommunikatbasen: „Kommunikatbasen bestehen aus solchen materialen Kommunikationsmitteln, die ein Kommunikationsteilnehmer produziert und die andere Kommunikationsteilnehmer aufgrund der Struktur ihres Wahrnehmungsapparates sowie durch Anwendung von regelhaften bzw. konventionalisierten Operationen als solche Gegenstände erkennen, denen sie Bedeutungen, Sinnbezüge und Relevanzen zuordnen und eventuell Handlungen als Konsequenzen daran anzuschließen gelernt haben.“ 


\section{Ikonische Sinnkonstitution - Boehm und Peirce}

Die Arbeiten des Philosophen und Kunsthistorikers Gottfried Boehm können in der aktuellen bildwissenschaftlichen Diskussion stellvertretend für eine Gruppe von theoretischen Ansätzen stehen, die man phänomenologisch nennen kann, insofern sie dafür eintreten, Bilder in erster Linie als Sichtbarkeitsgebilde, als visuell gestaltete Flächen, zu verstehen. Diese Ansätze und auch die bildtheoretischen Überlegungen von Boehm wurden in linguistischen Zusammenhängen allerdings bisher kaum aufgegriffen. Eine Anknüpfung wird an der Oberfläche dadurch erschwert, dass in phänomenologischen Ansätzen der Zeichenbegriff als bildtheoretischer Grundbegriff - zum Teil in schroffer Weise - abgelehnt wird. ${ }^{10}$ Bilder als Zeichen zu verstehen, reduziere diese entweder auf bloße Stellvertreter für eine bildunabhängige Sache ${ }^{11}$ oder unterstelle, in Analogie zur Sprache, eine arbiträre Relation von Ausdruck und Bedeutung sowie eine prädikative Struktur. Beides verstelle aber den Blick für die Besonderheit bildlicher Sinnkonstitution. „Bilder", so heißt es bei Boehm

besitzen eine eigene, nur ihnen zugehörige Logik. Unter Logik verstehen wir: die konsistente Erzeugung von Sinn aus genuin bildnerischen Mitteln. Und erläuternd fügen wir hinzu: diese Logik ist nicht-prädikativ, das heißt nicht nach dem Muster des Satzes oder anderer Sprachformen gebildet. Sie wird nicht gesprochen, sie wird wahrnehmend realisiert. (Boehm [2004] 2007b, S. 34)

Den Kern dieser Logik und damit auch seines bildtheoretischen Ansatzes bildet für Boehm eine spezifische Differen₹, die er „ikonische Differen₹" (ebd., S. 49) nennt - wobei ikonisch in seinen Texten konsequent mit bildlich zu übersetzen ist: Es ist die Differenz zwischen dem, was Betrachter bei der Betrachtung eines Bildes vor sich sehen, und dem, was sie darin (wieder-)erkennen. Boehm sieht im Vollziehen dieser Differenz ein Moment des ,Umspringens ${ }^{c}$ von Wahrnehmung in Sinn, das an außerbildliche Wahrnehmungserfahrungen anschließt, zugleich aber in charakteristischer Weise über diese hinausgeht. ${ }^{12}$

10 Diese Ablehnung durchzieht die philosophische Phänomenologie von ihren Anfängen an. In besonders zugespitzter Weise hat sie Lambert Wiesing (2005, bes. S. 30 f.) unter Rekurs auf Edmund Husserl noch einmal formuliert. Die Hürde, die damit für eine produktive linguistische Rezeption verbunden ist, zeigt sich indirekt etwa an der ebenfalls recht pauschalen Kritik Stöckls (2004a, S. 64 f. Anm. 28 und S. 92 f.), der den antisemiotischen Zügen phänomenologischer Ansätze seinerseits Inkonsequenzen vorwirft, ohne diese genauer aufzuzeigen. In erster Linie zieht er sich auf die Position zurück, dass diese Ansätze allenfalls für bestimmte Bildtypen besonders der künstlerischen Avantgarden Relevanz beanspruchen könnten, nicht aber für den massenmedialen Bildgebrauch.

11 Vgl. Boehm (1978, S. 453 f.): „Bilder sind nicht Substitute von Sachlagen, d.h. Sachlagen im Bild noch einmal, schon deshalb nicht, weil ,Sach'-Elemente des Bildes von der Weise ihres Erscheinens, Wirkens und Bedeutens nicht separiert werden können. Auch die Verwendung des semiotischen Zeichenbegriffs auf das Bild, die auf der Trennung von Signifikanten und transzendentem sprachförmigem Signifikat beruht, bricht aus eben diesen Gründen zusammen."

12 Die zentrale Rolle, die der Differenz als Ausgangspunkt für die Konstitution von Sinn gegeben wird, unterscheidet die Bildtheorie Boehms von solchen phänomenologischen Ansät- 
Was damit gemeint ist, lässt sich anhand der oben bereits erwähnten Doppelseite aus dem STERN (Abb. 2) nachvollziehen. Wenn man diese Seite ansieht, dann sieht man nicht einfach eine Fläche mit hellen und dunklen Flecken, sondern erkennt zugleich drei Fahrzeuge und wahrscheinlich noch einiges mehr. Außerdem sieht man sofort, dass es sich um die Seite aus einer Illustrierten handelt. Eine Antwort auf die Frage, wie Betrachter dazu kommen, in der schwarz-grau-weißen Fläche all dies zu erkennen, muss zwei Ebenen der Sinnkonstitution berücksichtigen und damit zwei Formen der Differenzbildung voneinander unterscheiden: ${ }^{13}$ eine Ebene, auf der die bildliche Sinnkonstitution eng auf außerbildliche Prozesse der Wahrnehmung bezogen ist und eine, durch die sie sich als bildliche von diesen unterscheidet.

Auf einer ersten Ebene kann man festhalten, dass die drei Fahrzeuge erkennbar werden, weil sie in der Wahrnehmung als bestimmte Figuren von einem unbestimmten Umfeld oder Grund unterschieden werden. Boehm beschreibt diesen Prozess folgendermaßen:

Wenn wir von Bildern reden (flachen, plastischen, technischen, räumlichen etc.), meinen wir eine Differenz, in der sich eine oder mehrere thematische Brennpunkte, die unsere Aufmerksamkeit binden, auf ein unthematisches Feld beziehen. (Boehm [2004] 2007b, S. 48 f.)

Auf das Beispiel bezogen: Damit Fahrzeuge erkannt werden können, muss die materielle Oberfläche des Bildes so gestaltet sein, dass sie entsprechende Unterscheidungen ermöglicht und sie dem Betrachter nahe legt. Sinnvoll werden diese allerdings nur, indem sie zum Anlass eines wiedererkennenden Sehens ${ }^{14}$ werden, bei dem die aktuell sichtbaren Kontraste einer Reihe früherer Seherfahrungen zugeordnet werden.

Diese sinnkonstituierende Zuordnung, die in der Regel unbewusst erfolgt, ist allerdings zunächst einmal noch nichts, das für Bilder spezifisch wäre. Denn in jedem optischen Wahrnehmungsprozess werden aktuellen Qualitäten im Wahrnehmungsfeld, wie etwa visuellen Kontrasten, Wahr-

zen, die im Rückbezug auf Husserl die Besonderheit des Bildes in einer spezifischen sichtbaren Präsenz sehen (vgl. insb. Wiesing 2005). Boehm selbst sieht Verbindungspunkte zum Saussureschen Sprachdenken (Boehm 1978, S. 460) und es ist dieser Grundzug der Sinnkonstitution durch Differenzen, der seine theoretischen Überlegungen bei aller Betonung der Eigenständigkeit von Bildphänomenen für den hier vertretenen pragmatischen Ansatz anschließbar macht.

13 Diese Unterscheidung ist bei Boehm angelegt, wird von ihm aber nicht immer explizit markiert.

14 Wiedererkennendes Sehen ist einer der Kernbegriffe der Ikonik Max Imdahls, einer kunstwissenschaftlichen Methodologie, die in ihren theoretischen Implikationen mit der Bildtheorie von Boehm über weite Strecken kompatibel ist - eine Kompatibilität, die einen biografischen Anhalt darin hat, dass Boehm nach einer philosophischen Promotion bei Hans-Georg Gadamer in Heidelberg als Assistent von Imdahl an der Bochumer Ruhr-Universität arbeitete. Jede ikonische (d.h. am Rahmen der Ikonik orientierte) Bildanalyse erfordert nach Imdahl eine Verbindung des „,wiedererkennende Gegenstandssehen[s]“ mit einem sehenden Sehen, das die „formale Ganzheitsstruktur“ eines Bildes als eines flächigen Objekts - seine „planimetrische Komposition“ - erschließt (Imdahl [1980] 1996, S. 487). 
nehmungsobjekte zugeordnet. Das setzt „Synthesen“ ${ }^{15}$ wie Boehm es unter Rückbezug auf Kant nennt, oder Schematisierungen voraus. Die Schematisierungen bestehen in Erfahrungen und Gewohnheiten, die das Sehen vororientieren. Und sie sind der Grund dafür, dass Peirce im Unterschied zur Phänomenologie Husserls ${ }^{16}$ den Wahrnehmungsprozess semiotisch versteht. Als wiedererkanntes Objekt ist der Gegenstand der Wahrnehmung gerade nicht unmittelbar sichtbar präsent, sondern nur vermittelt über Erfahrungen, die die Zuordnung regeln. Versteht man mit Peirce Symbole als Zeichen, die in einer solchen Regelmäßigkeit der Zuordnung begründet sind, die wie er an einer Stelle lapidar und zugleich konsequent pragmatistisch formuliert - ,mit ihren Bedeutungen durch ihre Verwendung verknüpft worden sind ${ }^{\text {" }}{ }^{17}$ dann wird deutlich, dass mit der Orientierung durch Erfahrung schon in der Semiose der Wahrnehmung ein symbolisches Moment wirksam ist. Es tritt bei Bildern noch stärker hervor. Allerdings handelt es sich auch bei gegenständlichen Bildern nicht um idealtypische Symbole. Denn wie in der außerbildlichen Wahrnehmung hängt die Wiedererkennbarkeit von Gegenständen im Bild nicht allein von dem etablierten Zuordnungsschema ab, sondern zugleich in hohem Maße von den Eigenschaften - von den Qualitäten - die aktuell sehend wahrgenommen werden. Es ist insofern für Peirce - wie für Boehm, dessen Terminologie sich hier mit der von Peirce berührt in der bildlichen wie auch in der außerbildichen Wahrnehmung ein ikonisches Moment wirksam. Denn die Begründung einer Zeichenrelation in Qualitäten des Repräsentamens bzw. Zeichenträgers ist für Ikone charakteristisch. Peirce bestimmt das Ikon idealtypisch als Zeichen, das „sich auf das von ihm denotierte Objekt lediglich aufgrund von Eigenschaften bezieht, die es selbst besitzt" ${ }^{\text {"18 }}$ In dieser Zuspitzung ist das Ikon die bloße Möglichkeit eines Zeichens, aber entscheidend an dieser Bestimmung ist die Betonung von Eigenschaften des Zeichenträgers als Ausgangspunkt von ,ikonischen ${ }^{6}$ Semiosen. Sie wird leicht dadurch verdeckt, dass Peirce im Zusammenhang mit seinen Definitionen des Ikon gerade in frühen Texten immer wieder auf eine Relation der Nachahmung oder auf die Ähnlichkeit zwischen Zeichenträger und Objekt abhebt. ${ }^{19}$ Geht man jedoch von der zitierten Defi-

15 „Die ikonische Differenz vergegenwärtigt eine visuelle Kontrastregel, in der zugleich ein Zusammensehen angelegt ist. Ikonische Synthesen sind bereits in der Struktur unserer Wahrnehmung angelegt.“" (Boehm [2004] 2007b, S. 49). Vgl. zum entsprechenden Begriff der „figürlichen Synthesis" sowie der Rolle von Begriffsschemata für die Wahrnehmung bei Kant den Beitrag von Lohmar (1993), der zudem - bei allen Differenzen der jeweiligen Ansatzpunkte - hinsichtlich der orientierenden Rolle von Erfahrungen deutliche Parallelen zwischen Kants Konzeption und Husserls Phänomenologie der Auffassung aufweist.

16 Vgl. etwa Husserl ([1913] 1992, \43).

17 Peirce (1986, S. 193 (Die Kunst des Räsonierens, MS 404, 1893)). Vgl. auch die folgende Definition: „Ein Symbol ist ein Zeichen, dessen zeichenkonstitutive Beschaffenheit ausschließlich in der Tatsache besteht, daß es so interpretiert werden wird.“ (Peirce 1983, S. 65).

18 Peirce (1983, S. 124 (Nomenklatur und Unterteilung der triadischen Relationen, 1903)).

19 Vgl. etwa Peirce (1986, S. 193 (Die Kunst des Räsonierens, MS 404, 1893) oder S. 205 (Kurze Logik, 1895)). 
nition aus, wird deutlich, dass Ähnlichkeitsurteile gerade nicht die selbstevidente Voraussetzung ikonischer Relationen sind. Sie sind vielmehr das Resultat von Semiosen, in denen jeweils durch gegebene Eigenschaften motivierte, ikonische Bezugsmöglichkeiten über eine Folge von direkten Zuordnungen, bei denen mit der notwendig konkreten Herstellung von Bezügen auch ein indexikalisches Verweismoment ins Spiel kommt, zur symbolischen Gewohnheit geworden sind. Diese Verbindung der drei Dimensionen zeigt auch die folgende Passage:

Wir sagen über ein Porträt einer Person, die wir nicht kennen, es sei überzeugend.
Soweit es mich bloß aufgrund dessen, was ich darin sehe, veranlaßt, mir eine Vor-
stellung von der dargestellten Person zu bilden, ist es ein Ikon. Aber in Wirklichkeit
ist es kein reines Ikon, weil ich weiß, daß ich stark von der Wirkung beeindruckt
bin, die - vermittelt über den Künstler - durch das Aussehen des Originals verur-
sacht wurde und also in einer genuin obsistenten Relation zum Original steht [als
solche bezeichnet Peirce hier den Index; Anm. d. Autors]. Nebenbei bemerkt, ich
weiß, daß Porträts nicht die leiseste Ähnlichkeit mit ihrem Original haben, außer in
bestimmten konventionellen Hinsichten und nach einer konventionellen Werte-
skala und so weiter. (Peirce 1986, S. 391 (Aus den Entwürfen für ein Logikbuch,
MS 425 A, 1902))

Wichtiger als der an dieser Stelle eher problematische Rekurs auf das indexikalische Moment der Semiose - das für den Umgang mit Porträts in der Tat wichtige Wissen um eine wirkliche Verbindung zwischen Porträtbild und Porträtiertem lässt sich kaum auf eine Verursachungsrelation reduzieren - ist die Betonung der Tatsache, dass sich der Ähnlichkeitseindruck immer bestimmten ,konventionellen Hinsichten' verdankt und dass sich die Überzeugungkraft eines Bildes mithin dem Zusammenspiel ikonischer und symbolischer Momente verdankt.

In dem Ausgehen von Eigenschaften liegt der Verbindungspunkt zwischen der Peirceschen Bestimmung ikonischer Zeichen und der phänomenologischen Bildtheorie Boehms. Anders als Peirce, dem es im Rahmen seiner philosophischen Semiotik nicht um eine Bildtheorie geht, betont Boehm allerdings neben den Gemeinsamkeiten zugleich den Unterschied zwischen außerbildlichen Wahrnehmungsprozessen und derjenigen Form einer Sinnkonstitution durch Differenzen, wie sie für Bilder charakteristisch ist. Die ikonische Differenz bezieht sich bei Boehm immer auch und in erster Linie auf diese zweite Ebene der Sinnkonstitution, die dadurch entsteht, dass bei Bildern sichtbare Kontraste auf einer begrenzten und überschaubaren Fläche fixiert sind. Das , unthematische Feld' der oben zitierten Passage, auf dem sich etwas erkennbar abzeichnet, hat nämlich beim Bild stets feste Grenzen. Das führt dazu, dass die einzelnen Teile, die als Figuren wiedererkannt werden können, nicht nur in einem sichtbaren Verhältnis zu ihrem relativ unbestimmten Umfeld stehen, sondern zugleich auch zur Bildfläche als einem - in seinen Grenzen wiederum bestimmten - Ganzen. Bilder sind 
- wie Boehm an einer anderen Stelle schreibt - „,nicht einfach geformte Dinge [...], sondern komplexe, dem Auge dargebotene Anordnungen, in denen dies oder jenes erscheint" (Boehm 1999, S. 170).

Entsprechend sind auch in dem Beispiel aus dem STERN nicht nur einfach drei Fahrzeuge und einige Figuren erkennbar, sondern sie sind zugleich in einer bestimmten Weise im Bild angeordnet, die sehend nachvollzogen werden kann. ${ }^{20} \mathrm{Zu}$ dieser Anordnung gehört die Tatsache, dass am unteren Bildrand eine Reihe von dunklen Silhouetten platziert sind, die den Betrachtern eine bestimmte Blickrichtung vorschlagen. ${ }^{21}$ Sie offerieren, dem Straßenverlauf, der mit der Bordsteinkante am linken Bildrand markiert wird, entlang der beiden vorderen Wagen in den Bildraum hinein zu folgen (Abb. 3).

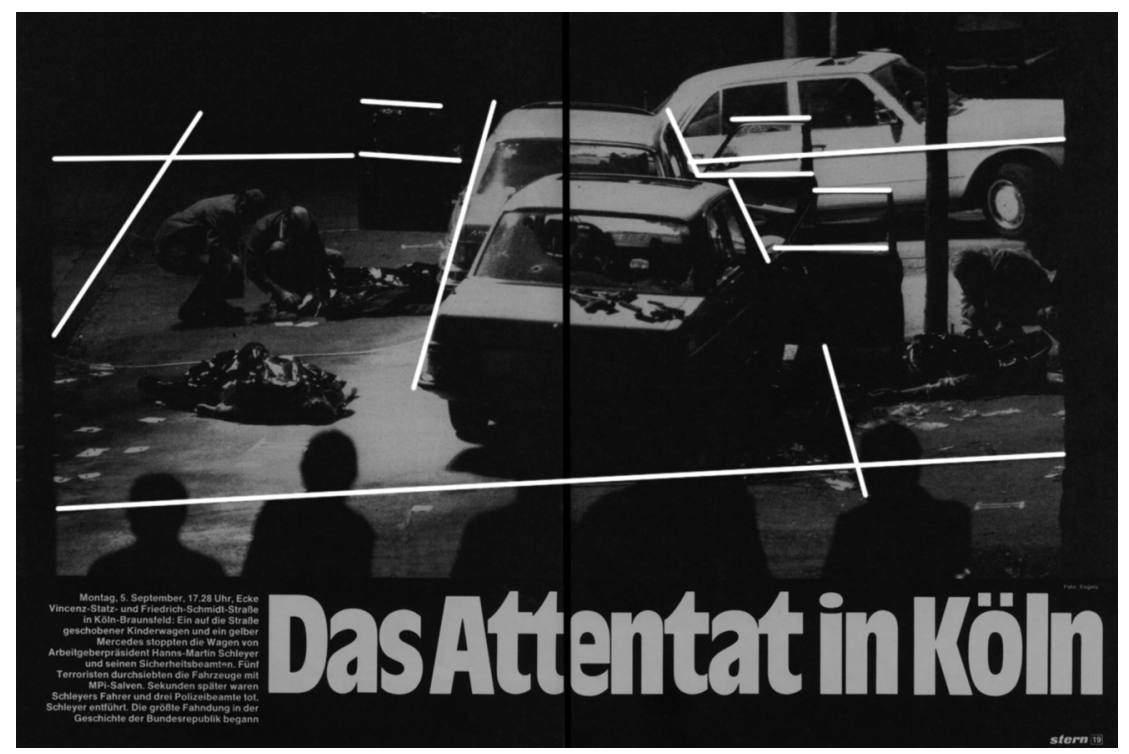

Abb. 3: Hervorhebung von Bildlinien

$20 \quad$ Für dieses sehende Sehen als Komplement zum wiedererkennenden Seben spielen in den Analysen Imdahls (siehe oben Anm. 14) immer wieder Bildlinien eine entscheidende Rolle.

21 Fehrmann/Linz (2008) führen verschiedene experimentelle Befunde dafür an, dass gerade ein abgewendeter Blick auch in statischen Bildern „das Mitvollziehen einer identifizierenden Bewegung herausfordert" (S. 280) und insofern bilddeiktisch funktioniert: Er wird als ,,als deiktischer Index rezipiert und damit von Betrachter - anders als der direkte, primär adressierende Blick - zu einer Verweishandlung vervollständigt" (S. 281). Das berührt sich mit Überlegungen bei Kemp ([1985] 1996, S. 247 ff.), der in seinen Analysen von Bildern aus dem Kanon der Kunstgeschichte allerdings zu einer etwas anderen Unterscheidung kommt. Er unterscheidet zwischen Rezeptionsvorgaben, wie sie Figuren machen, die den Betrachter direkt adressieren und auf einen Bildteil verweisen, und Rezeptionsangeboten durch Betrachterfiguren, die ihn eher vorsichtig auf etwas hinführen. 
Die durch diese Anordnung betonte Senkrechte wird im oberen Bilddrittel allerdings von der Waagerechten des dritten Wagens gekreuzt. Die Zierleiste des Wagens findet ihre bildliche Fortsetzung auf der linken Seite in der Grenze zum Schwarz des Hintergrundes. Unterstrichen wird diese Waagerechte durch die Konturen der aufgerissenen Türen an den vorderen Wagen, und schließlich noch durch eine feine Linie im unteren Bilddrittel, die anhand von anderen Bildern als Markierungsband der Spurensicherung identifiziert werden kann. So legt der Bildaufbau es dem Betrachter nahe, den Ereignisverlauf, in dem die Wagen von Schleyer und seinen Begleitern von den Attentätern abrupt zum Stillstand gebracht wurden, visuell nachzuvollziehen. Hinzu kommt, dass inmitten der Szenerie und fast genau in der Mitte der Bildfläche das Ereignis der tödlichen Schüsse in Form einer Kombination sichtbarer Spuren ins Bild gesetzt wird: Denn hier ist die zerstörte Heckscheibe des hinteren Wagens platziert und direkt darunter sind die zurückgelassenen Tatwaffen zu sehen, die auf dem Kofferraumdeckel liegen. Die Figuren im Vordergrund orientieren nicht nur den Blick, sondern unterstreichen, wenn man sie als spectators bzw. als Publikum versteht, auch die Spektakularität des Geschehenen. Die Fotografie ist damit mehr als ein dokumentarischer Beweis, der sich dem indexikalischen Charakter der fotografischen Technik verdankt. Durch die Anordnung der Elemente und die Ausrichtung an Bild-Linien wird sie zu einer visuell überzeugenden Veranschaulichung des „Attentats“ und seines Verlaufs. Die Pressefotografie, die erst nach der Tat entstanden ist, kann diesen Verlauf gerade nicht in einem direkten Sinne abbilden. Sie macht die Brutalität des Vorgehens aber in diesem Fall mit genuin bildlichen Mitteln nachvollziehbar.

Diese knappe Beispielanalyse kann andeuten, was es in Boehms Sinn heißt, dass mit der Fixierung und Positionierung sichtbarer Größen im Rahmen der Bildfläche neue Möglichkeiten der Sinnbildung entstehen. Sie resultieren daraus, dass die gestaltete Fläche als materielles Objekt mit bestimmten Eigenschaften - Farben, Kontrasten, usw. - angesehen werden kann und dass sie zugleich im sehenden Nachvollziehen ihrer Anordnung als Bild mit einem bestimmten Sinn verstanden werden kann. In dieser Gleichzeitigkeit von Sehen und Verstehen liegt für Boehm die Besonderheit bildlicher Sinnkonstitution. ${ }^{22}$ Die beständige Rückverwiesenheit des Sinns an die Qualitäten der Oberfläche ist aus seiner Sicht der Grund für die besondere Überzeugungskraft von Bildern, für ihre Evidenz (vgl. Boehm 2007a, S. 16).

22 Vgl. Boehm (1999, S. 170): „Wer den Gehalt eines jeweiligen Bildes [...] im Kontext anderer Bilder verstehen will, der muss sich auf Distinktionen einlassen, die er am jeweiligen Artefakt abliest. Abliest an der spezifischen Differenz, die mediale Prämissen und Formentscheidungen jeweils miteinander ausgebildet haben." 


\section{Die symbolische Dimension - Darstellung als schematisierte Anordnung}

Als konstitutiv für Boehms Konzept der ikonischen Differenz erweist sich ein Spannungsverhältnis zwischen dem schematisierten Wiedererkennen von wahrnehmungsbezogenen Bildbedeutungen und dem am einzelnen Bild sehend nachzuvollziehenden und darin visuell überzeugenden Bildsinn. Die Tendenz Boehms, dieses Spannungsverhältnis als eines zwischen stabilisierten Bedeutungen auf der Ebene von einzelnen wiedererkennbaren Bildteilen und einem hochgradig an die individuelle Bildanordnung gebundenen Sinn auf der Ebene des Bildes als Ganzem anzusiedeln, ist allerdings nicht ganz unproblematisch, wenn man weniger als der Kunsthistoriker Einzelbilder wie Gemälde, sondern den massenmedialen Gebrauch von Bildern analytisch in den Blick nimmt. Denn dann wird auch auf der Ebene des Bildes als Ganzem die Frage nach einer durch Schematisierungen des Gebrauchs stabilisierten, überindividuellen Bedeutung wichtig, für die Boehm sich allenfalls am Rand interessiert. Die folgende Grafik verdeutlicht diesen Zusammenhang.

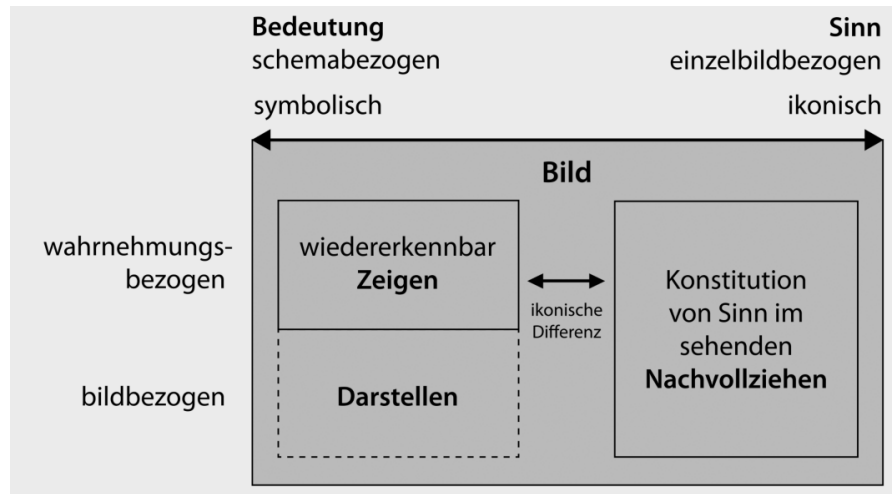

Grafik 1: Bezugsgrößen der bildlichen Sinnkonstitution

Um auch terminologisch zu unterscheiden: Es geht nicht nur darum, was Teile eines Bildes wiedererkennbar zeigen, sondern was es als Ganzes erwartbar darstellt. Das Problem lässt sicht erneut am Beispiel aus dem STERN verdeutlichen. In dem Bild sind nämlich nicht nur Autos und andere Dinge erkennbar, es ist auch ein Tatort zu sehen. Er ist kein weiterer Gegenstand, den ein Teil des Bildes wiedererkennbar zeigt, sondern ein Sachverhalt. Das Sehen dieses Sachverhalts liegt auf einer anderen Ebene. Denn um einen Tatort zu sehen reicht es nicht, einfach die einzelnen gezeigten Gegenstände wiederzuerkennen, zusätzlich braucht es eine Kenntnis davon, wie ein Tatort - der Ort eines kurze Zeit zurückliegenden Gewaltgeschehens - üblicherweise bildlich dargestellt wird. Um ohne weitere Hinweise in dem Bild 

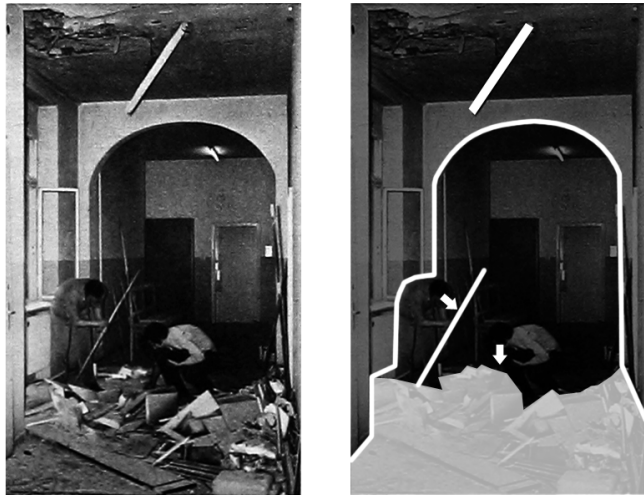

Abb. 4: QUICK 23, 31.5.1972, S. 16 (Ausschnitt)
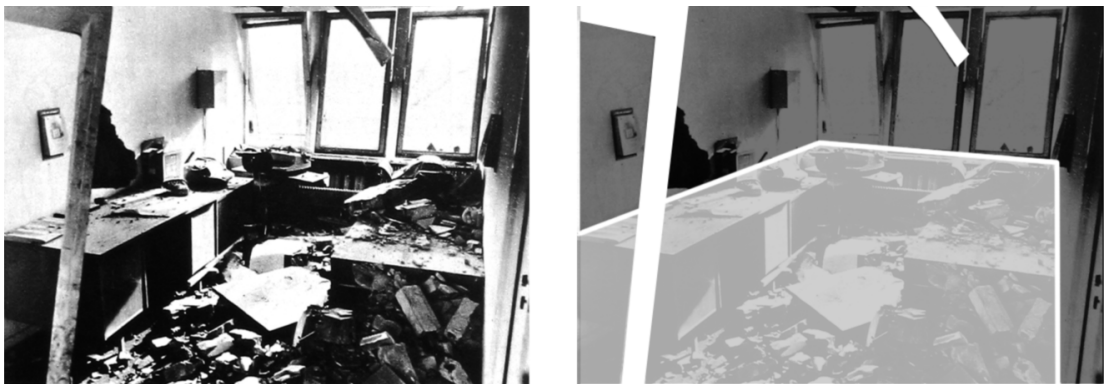

Abb. 5: SPIEGEL 23, 29.5.1972, S. 24 (Ausschnitt)
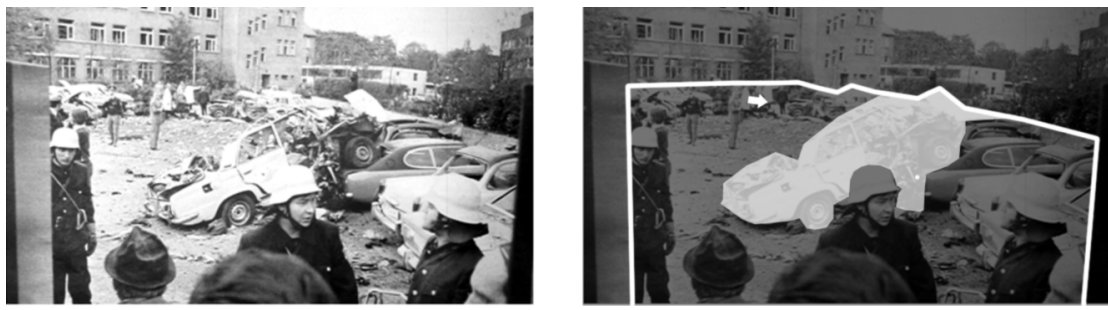

Abb. 6: STERN 23, 28.5.1972, S. 154 (Ausschnitt)
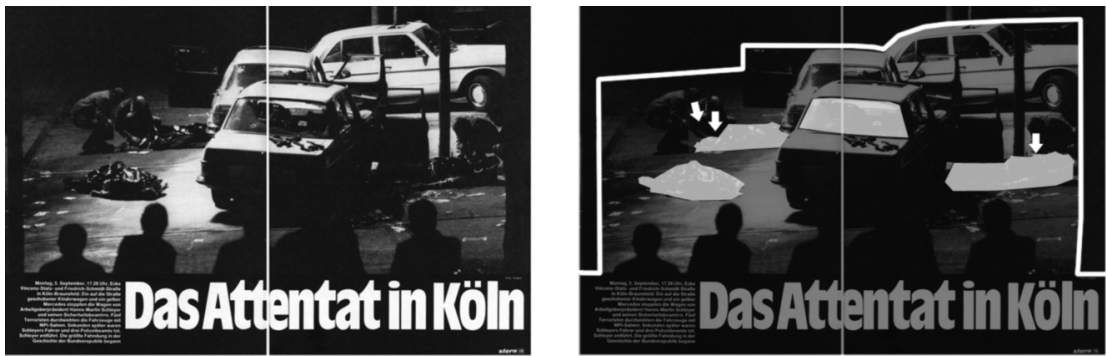

Abb. 7: STERN 38, 8.9.1977, S. 18-19, jeweils links Original rechts mit Markierungen 
einen Tatort zu sehen, müssen Betrachter in der Erfahrung mit anderen Bildern ein mehr oder weniger flexibles Schema aufgebaut haben, das es ihnen erlaubt, das Bild in eine Reihe mit diesen anderen Bildern zu stellen und so als Darstellung eines Tatorts zu verstehen.

Anhand einer entsprechenden Reihe von Bildern lassen sich daher auch rekonstruktiv die bildlichen Bestandteile eines solchen Tatort-Schemas gewinnen (Abb. 4, 5, 6, 7):

- Zunächst einmal wird durch einige Teile des Bildes eine räumliche Situation angedeutet, damit ein Ort erkennbar werden kann. Die Bilder von den Orten der Anschläge auf das Hamburger Springer-Haus (Abb. 4) und das Polizeipräsidium im Augsburg (Abb. 5) zeigen durch Wandfluchten links und rechts (sowie in einem Fall auch eine Deckenflucht am oberen Bildrand), die auf eine Stirnwand im Hintergrund zulaufen, einen Raum als Tatort an: Die Wände umschließen nach drei Seiten die Bodenfläche eines Innenraumes, der sich zum Betrachter hin öffnet. Der Blick auf die beiden weiteren Bilder kann zeigen, dass diese bildliche Anordnung, die an einen Bühnenraum denken lässt, nicht einfach das zufällige Nebenprodukt des Fotografierens in Innenräumen ist. Denn die Rahmung einer Fläche nach links und rechts sowie zum Hintergrund hin findet sich in abgewandelter Form auch hier.

- An den so markierten Orten werden als weiterer Bildbestandteil Gegenstände platziert, an denen Spuren der Tat erkennbar sind. Besonders deutlich ist dies beim Bild des Münchener Anschlags, bei dem auf der gerahmten ,Bühnenfläche' ein Fahrzeug mit deutlichen Zerstörungsspuren zu sehen ist, das zugleich im Zentrum der gesamten Bildfläche steht. Zerstörungsspuren finden sich in den Innenraumbildern aber auch an den rahmenden Teilen; auffällig sind insbesondere Teile der Deckenverkleidung, die von oben schräg in den Raum hineinreichen. Sie fallen gleichsam , aus dem Rahmen', ebenso wie die Fensterflügel im Vorder- und Hintergrund des SPIEGEL-Bildes. Eine solche schräge Positionierung im Bild, die als Abweichung von der üblichen Ausrichtung der Gegenstände die Aufmerksamkeit auf sich zieht, findet sich schließlich auch bei dem zerstörten Fahrzeug des Bildes aus München.

- Die prominente - meist zentrale - oder ungewöhnliche - in den Beispielen zumeist schräge - Positionierung der Gegenstände im Bild trägt bereits dazu bei, die Aufmerksamkeit des Betrachters auf die Folgen der Gewaltanwendung als Hauptbestandteil des Tatort-Motivs zu lenken. Ein weiteres bilddeiktisches Mittel, das für Tatortbilder typisch ist, sind die bereits erwähnten Betrachterfiguren im Bild. Das sind neben Personen in Rückenansicht am unteren Bildrand, die als Zuschauer am Ort des Geschehens den Betrachterblick in den ,Bühnenraum‘ hinein lenken, auch Personen, die zwischen den zerstörten Gegenständen platziert sind und deren Blick auf die Spuren gerichtet ist. 
Keiner der Bestandteile ist in der vorgeführten Form unersetzlich für eine Tatortdarstellung, aber zusammen bilden sie eine prototypische Konstellation. Diese Konstellation ist allerdings nicht mehr einfach ein Schema der außerbildlichen Wahrnehmung, sondern eine bildbezogene Größe - eine schematisierte Verbindung von wiedererkennbaren Elementen in ihren üblichen Anordnungen auf der Bildfläche. Sie kann als Motiv bzw. Motivtyp oder eben als Darstellungsschema bezeichnet werden. Die Schematisierung und ihre Verbindung mit einer Darstellungsbedeutung ist ein Produkt des Bildgebrauchs und hat damit nach Peirce symbolischen Charakter. Doch sind es erneut die variablen sichtbaren Eigenschaften der einzelnen Bilder, die es erlauben, sie in eine Reihe von Tatort-Darstellungen zu stellen. Auch auf der Ebene der Darstellung verbindet sich also die symbolische Dimension in der Semiose mit der ikonischen, die dafür sorgt, dass die jeweiligen Einzelbilder immer auch mehr oder weniger typische Vertreter des motivischen Paradigmas sind. In der obigen Grafik ist diese Gleichzeitigkeit der beiden Dimensionen in Form einer polaren Skala berücksichtigt. Deren Endpunkte sind durch die idealtypischen Peirceschen Bestimmungen des Ikons und des Symbols definiert, die als Reinformen gerade nicht vorkommen. Die skalare Darstellung hält jedoch fest, dass die Semiose stärker durch den Bezug auf ein Schema geprägt und damit ,symbolischer' sein kann. Das resultiert dann in relativ stabilen Bedeutungen - sei es auf der auf die außerbildliche Wahrnehmung bezogenen Ebene des wiedererkennbaren Zeigens oder auf der bildbezogenen Ebene der Darstellung. Die Semiose kann umgekehrt aber auch ,ikonischer' sein, wenn sie stärker an den wahrnehmbaren Eigenschaften des einzelnen Bildes und ihrem Beitrag zur Sinnbildung orientiert ist, für die sich Boehm in erster Linie interessiert. Das Ergebnis ist in diesem Fall ein jeweils neu vom Einzelbild her zu gewinnender und daher kommunikativ weit weniger gut stabilisierbarer Sinn.

\section{Prägnanz in Text-Bild-Zusammenstellungen}

Die bisherige Rekonstruktion der Darstellungsbedeutung von Bildern ist allerdings noch unvollständig. Denn ein Betrachter, der das STERN-Bild gemäß dem Tatort-Schema versteht, kann nicht nur sehen, sondern auch sagen, dass es einen Tatort darstellt. Diese Kopplung ist kein Zufall. Der Darstellungsbedeutung liegt nämlich immer schon eine Beziehung von Text und Bild zugrunde. Als situationsübergreifend erwartbare Größe resultiert die Bedeutung nicht allein aus einem wiederholten Bildgebrauch, sondern auch daraus, dass vergleichbaren Bildern bei deren Verwendung immer wieder ein entsprechender Darstellungs-Sinn durch Textteile zugeschrieben wurde. ${ }^{23}$ Zwei Beispiele, bei denen leichte Varianten des STERN-Fotos ver-

\footnotetext{
$23 \quad$ Im Fall von Printmedien ist hierbei in erster Linie an Bildunterschriften zu denken, doch
} auch die Haupt- und Zwischenüberschriften der Text-Beiträge können allein aufgrund ihrer 
wendet werden, können dies belegen (Abb. 8, 9). Das erste stammt aus einer rückblickenden Darstellung der Schleyer-Entführung in Buchform, die von den zwei STERN-Journalisten Kai Hermann und Peter Koch noch 1977 unter dem Titel Entscheidung in Mogadischu veröffentlicht wurde (Hermann/ Koch 1977, S. 6-7). Der oben links ins Bild gesetzte Textteil beginnt mit der Zeile „Der Tatort:“ Abgesetzt durch den Doppelpunkt schließt sich in den folgenden Zeilen eine genauere Lokalisierung des Ortes und die Einordnung einzelner Bildteile in den Ereigniszusammenhang an. Sie werden durch diese Zuschreibung zu Elementen der Tatortdarstellung erklärt: „die Vincenz-Statz-Straße im Kölner Villenvorort Braunsfeld. Vorn das Fahrzeug der Wachmannschaft, dann der Wagen Schleyers und hinten das von den Terroristen zur Hälfte quer über die Fahrbahn gefahrene Auto. Die toten Opfer des Überfalls sind mit Plastikplanen zugedeckt. Kriminalbeamte sichern Spuren“. Das zweite Beispiel stammt aus einem SPIEGEL-Heft des vergangenen Jahres (H. 37, 8.9.2008. S. 43), in dem in einer Titelgeschichte zum Kino-Film Der Baader-Meinhof-Komplex den Bildern von Filmszenen Pressefotos der Ereignisse gegenüberstellt wurden. Auch hier erklärt der ins Bild gesetzte Textteil das Foto zu einem Bild des „Tatort[s] der SchleyerEntführung in Köln 1977“.

Solche Beispiele ließen sich leicht vermehren, doch schon diese Auswahl macht deutlich: Darstellungsbedeutungen werden nicht allein durch vergleichbare bildliche Anordnungen konstituiert, sondern werden im kommunikativen Gebrauch von Text-Bild-Zusammenstellungen stabilisiert. Sie entstehen - so kann man unter Rückgriff Ludwig Jägers Konzeption kultureller Semantik formulieren - in intermedialen Transkriptionen (vgl. Jäger 2002, S. 29 ff.). Bestimmte Aspekte eines bildlichen Zeichens bekommen durch die sprachliche Transkription den Status von Skripten, sie werden durch die auf sie bezogenen Textteile lesbar gemacht. Das kann auf der Ebene des wiedererkennbaren Zeigens oder der Ebene des Darstellens geschehen. Doch das Bild ${ }^{24}$ wird durch diese Transkription gerade nicht ersetzt. Es bleibt eine gleichzeitig sichtbare Größe, auf die sich das schriftliche Transkript stützt. Ein entsprechender Textteil transkribiert also nicht nur das Bild, schreibt ihm aktuell einen bestimmten Sinn zu und trägt damit vielleicht im Diskurszusammenhang dazu bei eine Darstellungsbedeutung zu stabilisieren. Er setzt im gleichen Moment darauf, dass dieser Sinn in der Betrachtung des Bildes auch aktuell erfahrbar wird.

Position auf der Seite immer wieder als Zuschreibungen verstanden werden. Darüber hinaus nehmen Überschriften oder weitere Textpassagen in einigen Fällen explizit (etwa über deiktische Ausdrücke), häufiger allerdings implizit (über Beschreibungen von Objekten oder Sachverhalten, die parallel bildlich dargestellt - und in den Bildunterschriften benannt - werden) auf die abgedruckten Bilder Bezug, und werden so als Zuschreibungen lesbar.

24 Als Prätext bzw. Präskript in der Terminologie Jägers, die allerdings mit ihrer schriftsprachlichen Metaphorik an dieser Stelle, die das semiotische Material diesseits des intermedialen Transkriptionsvorgangs bezeichnet, eine linguistisch zwar verbreitete, aber deswegen nicht minder problematische Analogie von Text und Bild stützt (vgl. Steinseifer 2009). 


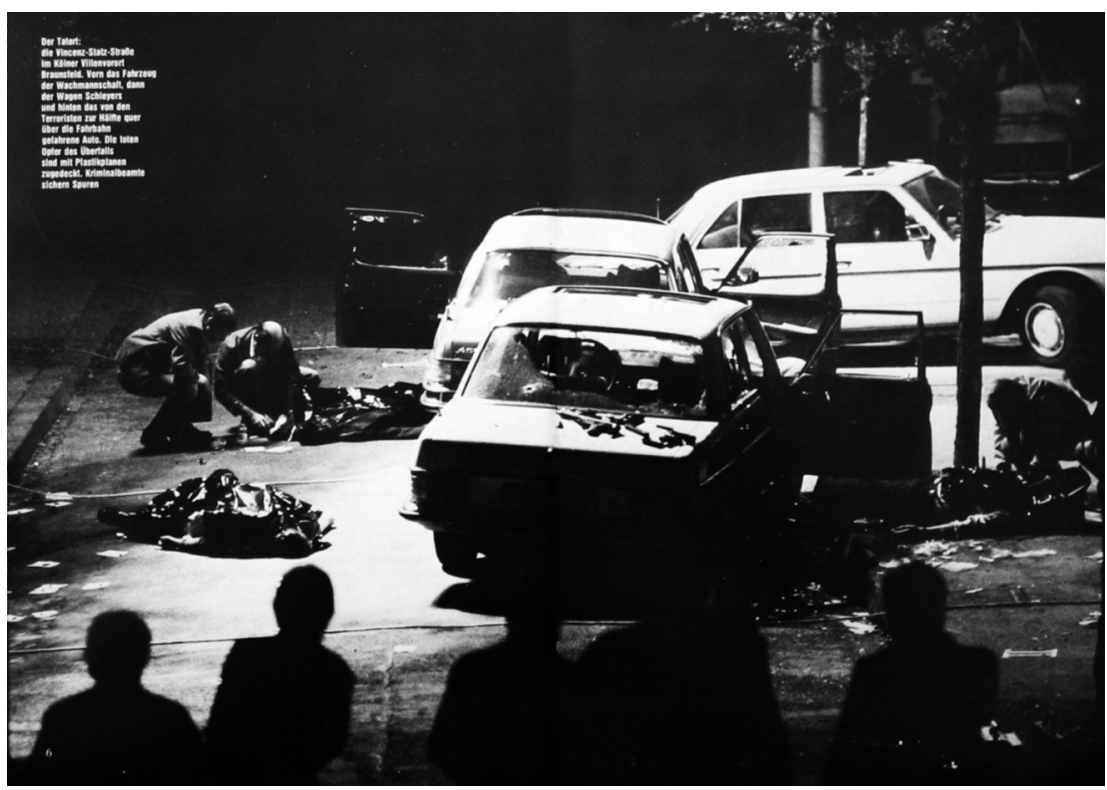

Abb. 8: Hermann/Koch 1977, S. 6-7

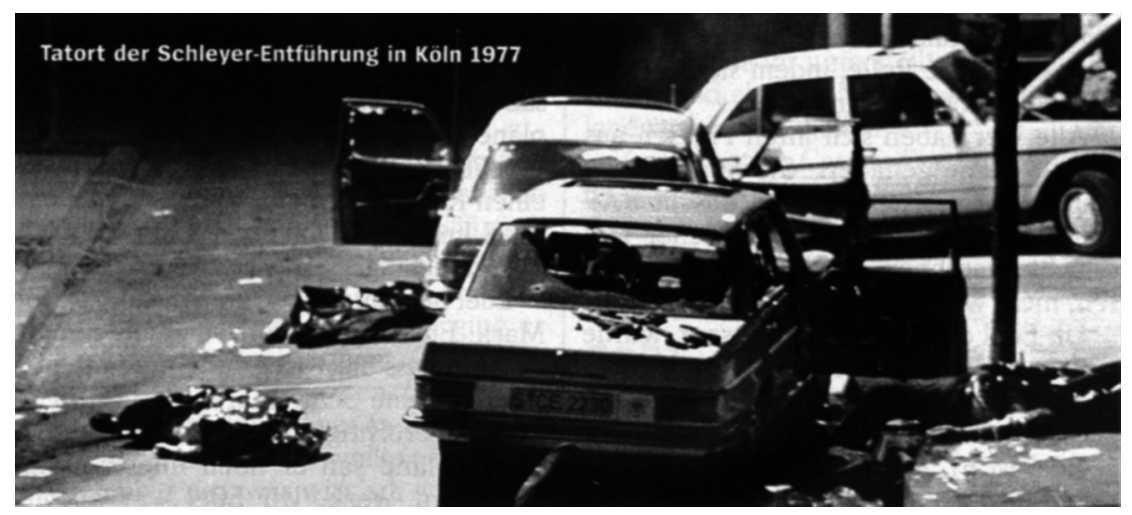

Abb. 9: SPIEGEL 37, 8.9.2008. S. 43 (Ausschnitt)

Dieser umgekehrte Zusammenhang deutet sich bei Jäger an, wenn er davon ausgeht, dass die Skripte, obwohl sie erst in der Transkription konstituiert werden, doch zugleich einen gegenüber dieser autonomen Status bekommen. In Bezug auf Bilder schreibt er, dass ,es gerade die Verwendung der diskursiven Sprache als Transkriptionsmedium ist, die das Bild als Bild in einen interventionsfähigen Skript Status versetzt" (Jäger 2002, S. 34). Die Begründung, die er anschließend gibt, - „deshalb nämlich, weil die diskursive Beschreibungssprache im Hinblick auf die Bildlektüre, die sie vor- 
schlägt, wahrheitswertfähig und insofern hinsichtlich ihrer Angemessenheit befragbar ist." - ist dann aber doch sehr stark von der sprachlichen Seite und dem Geschäft des philosophisch interessierten Interpreten her gedacht. Im massenmedialen Alltag der Vertextung von Bildern und ihrer Rezeption sind Konstellationen, die eine Intervention gegen deutende Zuschreibungen provozieren, selten. Doch auch in den Fällen, in denen eine Angemessenheit der schriftlichen Transkription zu konstatieren ist, hat die gleichzeitige Sichtbarkeit des Bildes, seine mediale Autonomie, einen Effekt. Insofern sie nämlich den zugeschriebenen Sinn nicht zurückweist, sorgt die zusätzliche Anwesenheit der bildlichen Zeichenform dafür, dass die Transkription plausibel erscheint. Die Zusammenstellung mit dem Bild kann so dem sprachlich artikulierten Sinn eine besondere, zusätzliche Überzeugungskraft bzw. Evidenz verleihen. ${ }^{25}$

Diesen Fall von Evidenz, der in der konkreten Text-Bild-Zusammenstellung wirksam wird, bezeichne ich als Prägnan₹. Ich wähle damit bewusst eine Bezeichnung, die linguistisch nur wenig vorbelastet ist, ${ }^{26}$ gleichwohl aber nicht ohne einschlägige Vorgeschichte. Denn Prägnanz ist ein Grundbegriff der Gestalttheorie (vgl. Hüppe 1984), jener zu Beginn des 20. Jahrhunderts entstandenen Richtung der Psychologie, die davon ausgeht, dass die Wahrnehmung immer durch Gestalten orientiert ist, durch phänomenale Ganzheiten, auf die einzelne Wahrnehmungsreize bezogen werden. Die Tendenz, Reizkonstellationen entlang solcher prägnanten Gestalten zu ordnen, konnte in Wahrnehmungsexperimenten mit einfachen geometrischen Formen empirisch bestätigt werden. ${ }^{27}$ Gleichzeitig konnten jedoch die Versuche, die so ausgezeichneten, guten Gestalten' durch Kriterien wie Regelmäßigkeit, Einfachheit bzw. Komplexität oder Symmetrie zu bestimmen, um durch Gestaltgesetze Prägnanzwirkungen vorhersagen zu können, nicht überzeugen, so dass die formalen Kriterien selbst bei einfachen geomet-

25 Nach Jägers Klassifikation handelt es sich um einen Fall von epistemischer Eviden₹ bei der „das semiotische Verfahren, dem sie sich verdankt, unter bestimmten Bedingungen selber unsichtbar bleibt, weil es hinter seiner Evidenz erzeugenden Wirkung verschwindet" (2008, S. 313).

26 Eine Ausnahme ist die Verwendung in der Textverständlichkeitsforschung. In Anlehnung an instruktionspsychologische Arbeiten der 1970er und 1980er Jahre wird mit Prägnanz hier das Verhältnis von semantischer Struktur und dem Umfang der zu ihrer Artikulation verwendeten Zeichen bezeichnet, wobei sowohl eine in Bezug auf eine bestimmte Textfunktion minimal komplexe semantische Struktur als auch ein in Bezug auf diese Struktur minimaler Zeichenaufwand als ökonomisch und damit prägnant gilt (vgl. zusammenfassend Göpferich 2008, bes. S. 303).

27 So konnte z.B. gezeigt werden, dass die Wahrnehmung eines Winkels, dessen Öffnungsgrad kontinuierlich verändert wird, etwa ab einem Öffnungsgrad von $85^{\circ}$ bis zu einem Öffnungsgrad von $95^{\circ}$ als ,ungefähr Rechter Winkel ${ }^{*}$ wahrgenommen wird, und ab einem Öffnungsgrad von $175^{\circ}$ als , leicht abgeknickte Gerade'. Bezugsgrößen wie spitzer, rechter, stumpfer Winkel und Gerade werden daher als Prägnanzstufen bezeichnet (vgl. für weitere Beispiele Hüppe 1984, S. 11 ff.). 
rischen Phänomenen um nicht-formalisierbare semantische Kriterien wie eine vorgeblich größere Bedeutungsfülle der , guten Gestalt' ergänzt wurden (ebd., S. 22 ff.). Diese Einsicht der Gestalttheorie in die Verschränkung von Wahrnehmungseindrücken und Bedeutungszusammenhängen verweist erneut auf die notwendige Verbindung der ikonischen und symbolischen Dimension in der Analyse von Zeichenprozessen. Die Verschränkung von Wahrnehmung und Bedeutung prägte auch die Übernahme des Prägnanzbegriffs in Ernst Cassirers Entwurf einer Philosopbie der symbolischen Formen. Wenn Cassirer unter symbolischer Prägnanz, „die Art [versteht], in der ein Wahrnehmungserlebnis, als ,sinnliches ${ }^{6}$ Erlebnis, zugleich einen bestimmten nicht-anschaulichen Sinn in sich fasst und ihn zur unmittelbaren konkreten Darstellung bringt" (Cassirer [1929] 1954, S. 235), dann verweist der nicht-anschauliche Sinn auf die in der Wahrnehmung immer schon vollzogene, durch die Erfahrung vermittelte Orientierung auf ein Ganzes, das nicht in der Summe der wahrgenommenen Teile und ihrer materiellen Eigenschaften aufgeht. Erfahrung versteht dabei auch Cassier als Form der symbolischen Synthese. Sie ist ein „theoretisches Gefüge“", das allerdings wiederum nur in der Verbindung mit der „konkreten Fülle“ materieller Qualitäten für Prägnanzeffekte sorgt.

Die ,Teilhabe‘ an diesem Gefüge gibt der Erscheinung erst ihre objektive Wirklichkeit und ihre objektive Bestimmtheit. Die ,symbolische Prägnanz', die sie gewinnt, entzieht ihr nichts von ihrer konkreten Fülle; aber sie bildet zugleich die Gewähr dafür, daß diese Fülle nicht einfach verströmt, sondern sich zu einer festen, in sich geschlossenen Form rundet. (ebd., S. 237)

Die hier vorgeschlagene Bestimmung von Prägnanz nimmt die Formorientierung der Gestalttheorie ebenso auf, wie die Verbindung von Gestalt und Gehalt - von Formeigenschaften mit einem Sinn -, ist aber deutlich spezifischer als diejenige Cassirers. Sie reserviert den Terminus für den Fall, dass ein gegenständliches Bild dem, was es in einem immer auch durch Texte konstituierten Diskurs-Zusammenhang mehr oder weniger erwartbar darstellt, zusätzliche Plausibilität verleiht. Je klarer einzelne Textteile in einer Text-Bild-Zusammenstellung - aufgrund ihrer Position oder aufgrund dessen, was sie sprachlich artikulieren - transkriptiv auf ein Bild oder mehrere Bilder zu beziehen sind, umso mehr profitieren sie umgekehrt von Prägnanzeffekten, die von wahrnehmbaren Eigenschaften der Zeichenform der entsprechenden Bilder ausgehen.

Was damit als Prägnanz bezeichnet wird, ist zwar auf den Sinn des jeweiligen Bildes bezogen, es handelt sich aber nicht einfach um eine weitere Stufe der Bedeutung oder der intermedialen Transkription. Die Prägnanz gehört als Evidenzeffekt, der von den sichtbaren Qualitäten des Bildes ausgeht, vielmehr zu den medienspezifischen Bedingungen transkriptiver Prozesse, in denen Bedeutungen fassbar werden. Dabei kommen ikonische 
und symbolische Momente zusammen, indem materielle Eigenschaften der Bildfläche zu Impulsgebern der Semiose werden und auch eine bereits etablierte Bedeutung immer wieder neu stützen. Die obige Grafik ist dann um diese Wechselbeziehung zwischen Bild und Text zu ergänzen. Der Pfeil für die Zuschreibung an das Bild steht dabei dem symbolischen Pol näher, nicht nur da die Schriftzeichen der Texte selbst dominant symbolischen Charakter haben, sondern auch - und in erster Linie - da sie zur symbolischen Stabilisierung der Bildbedeutung beitragen. Der gegenläufige Pfeil für den Prägnanzeffekt des Bildes steht dem ikonischen Pol näher, da die Überzeugungskraft von medienspezifischen Qualitäten ausgeht, von der sichtbaren Form, in der das Bild einen Sinn artikuliert.

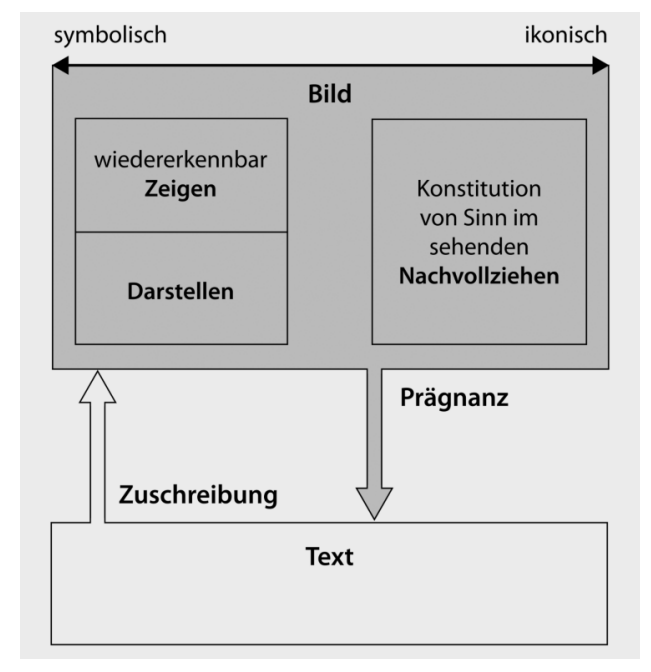

Grafik 2: Der Ort des Prägnanzeffekts in der TextBild-Zusammenstellung

Der Pfeil steht aber mit Absicht eher in der Mitte. Denn in Bezug auf die beiden Pole der Skala lassen sich wiederum idealtypisch zwei Varianten von Prägnanzeffekten unterscheiden. Das Unterscheidungskriterium ist, ob es sich - im Boehmschen Sinn - um die Evidenz eines einzelnen Bildes mit seiner besonderen Anordnung handelt, oder ob von einer bildlichen Anordnung ein Prägnanzeffekt ausgeht, weil sie als konkrete sichtbare Form zugleich ins Schema passt, das heißt: weil sie zum prototypischen Kernbereich der Varianten eins Motivtyps zu zählen ist. Der Unterschied zwischen beiden Varianten lässt sich erneut an den bereits diskutierten Beispielen von Text-Bild-Zusammenstellungen erläutern.

Bei der Text-Bild-Zusammenstellung der SPIEGEL-Titelseite kommt es für einen Prägnanzeffekt zunächst einmal darauf an, dass die ,Bomben in der Bundesrepublik', von denen die Titelzeile handelt, im verwendeten 
Bild erkannt werden können. Dazu wird hier eine bildliche Form gewählt, die typischerweise verwendet wird, um mit einem Bild darzustellen, wie das Gezeigte aussieht: Die thematischen Figuren sind im Zentrum platziert und heben sich vor einem einfarbigen, in diesem Fall orange-roten Hintergrund ab, der den Rest der Bildfläche einnimmt. Bilder, die gemäß diesem weitgehend themenunspezifischen Darstellungsschema aufgebaut sind, finden sich typischerweise in Warenkatalogen oder als Personendarstellungen im Bereich der Bewerbungsfotos. Aber auch im Artikel zur „Bomben in der Bundesrepublik"-Titelseite finden sich weitere entsprechende Darstellungen von „selbstgebastelten Sprengkörper[n]“ (SPIEGEL 23, 29.5.1972, S. 29, Abb. 10), die nicht nur ein Bild davon vermitteln, wie die gezeigten Molotow-Cocktails und Brandbomben aussehen, sondern als prägnante Darstellungen dieser Objekte den Berichten über die Eskalation der Gewalt durch die „,Stadtguerilleros ““ in Gestalt der „Baader-Meinhof-Gruppe“ zusätzlich Nachdruck verleihen.$^{28} \mathrm{Da}$ diese erste Variante von Prägnanzeffekten eng mit dem Erkennen eines Bild-Schemas und seiner Bedeutung - hier dem Darstellen des Aussehens - verbunden ist, kann man auch von schematischer Prägnanz. sprechen. Mit der Dominanz des Schemabezugs hängt auch der befremdliche Effekt zusammen, der im Fall der SPIEGEL-Titelseite entsteht, wenn man den Text der Titelzeile entfernt. Dann sind nämlich kaum noch Bomben zu erkennen. Da sich das Darstellungsschema nämlich dadurch auszeichnet, dass die Figur-Grund-Unterscheidung extrem betont wird, liefert das Bild keine weiteren Verstehenshinweise, sondern setzt voraus, dass man weiß, was das Bild zeigt, dass man den Gegenstand, dessen Aussehen dargestellt wird, wiedererkennen kann. Wenn man für den gezeigten Gegenstand noch kein wahrnehmungsbezogenes Schema ausgebildet hat, gelingt dies nur dank der benennenden Zuschreibung. Daran wird erneut deutlich, dass die Prägnanz, obwohl sie mit wahrnehmbaren Eigenschaften der bildlichen Ausdrucksform zusammenhängt, selbst keine Eigenschaft des Bildes ist, sondern ein inter-medialer Effekt der Bildverwendung.

28 Die Selbstbezeichnungen „Stadtguerrilla“ und „Stadtguerillero“ werden in dem Artikel in distanzierende Anführungszeichen gesetzt, während „Baader-Meinhof-Gruppe“ die im SPIEGEL zu dieser Zeit übliche Fremdbezeichnung für die Rote Armee Fraktion (RAF) ist. Über die für den Terrorismus-Zusammenhang typische Annahme einer Eskalation der Gewalt wird im Artikel des SPIEGEL der Bezug zur Studentenbewegung hergestellt: ,Was einst an Deutschlands Hochschulen als Massenaufstand gegen überkommene Autoritäten und für eine bessere Welt begonnen, die ,Außerparlamentarische Opposition“ (Apo) auf den langen Marsch durch die Institutionen gebracht, Tausende zu Knüppelorgien und Steineschlachten auf die Straße getrieben hatte, mündet endgültig im kriminellen Untergrund. Was mit Transparenten (,Haut den Springer auf die Finger), mit Pudding-, Tomaten-, und Eierwürfen begann, mit Molotow-Cocktails und Brandschatzung (,Burn, warehouse, burn') eskalierte, ist jetzt bei Raub, Mord und Totschlag angekommen." (SPIEGEL 23, 29.5.1972, S. 24-34, hier S. 25). 


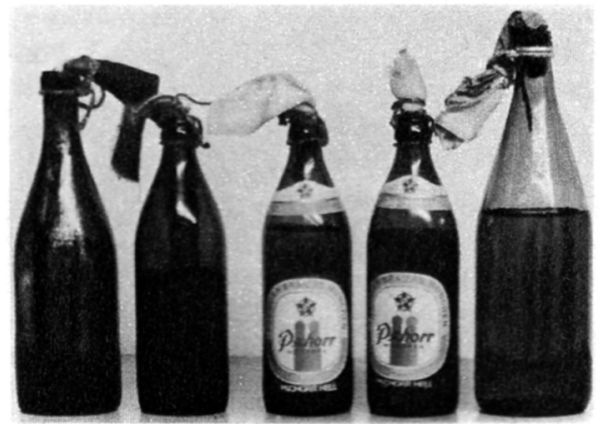

Molotow-Cocktail (Münchner Altersheim)

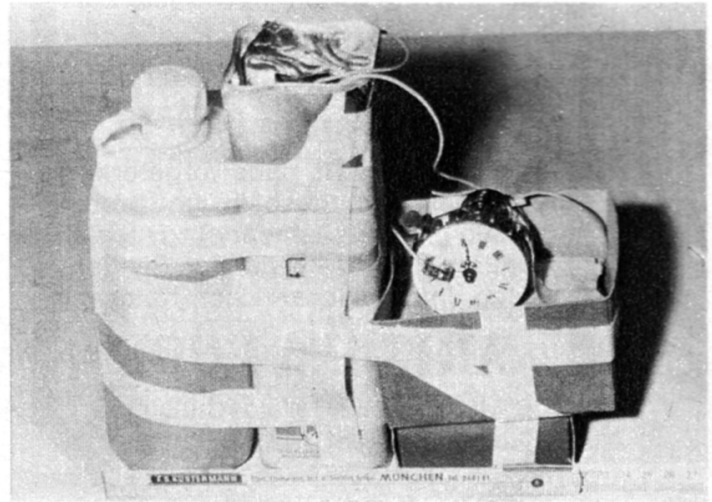

Brandbombe (Münchner Justizgebäude)

Abb. 10: SPIEGEL 23, 29.5.1972, S. 29

Der Vergleich der Bilder des Artikels mit der Titelseite macht neben dem Schema allerdings auch eine Besonderheit des Titelbildes deutlich. Auf dem Titel wird nämlich durch die Art, wie die Gegenstände im Bildfeld positioniert sind, nicht nur das Aussehen der Bomben dargestellt. Zugleich wird auch noch ihre Größe betont, indem sie über den weißen Rahmen, der üblicherweise das Titelbild begrenzt, hinausreichen, den SPIEGEL-Schriftzug überlagern und rechts an den Rand der Bildfläche stoßen. Diese Betonung der Größe erfolgt mit bildlichen Mitteln, die ebenso konventionell sind, wie sie nur anhand der jeweils sichtbaren Konstellation nachvollziehbar werden. Man kann diese Positionierung im Bild als Betonung der Bedrohung, die von den ,Bomben in der Bundesrepublik ${ }^{6}$ ausgeht, verstehen: Sie sprengen gleichsam den Rahmen. Und man kommt dann in den Bereich der zweiten ikonischen Variante der Prägnanz. Man muss das Bild aber nicht so verstehen, damit es im Bezug auf den Text qua Schema einen verstärkenden Effekt hat und für Prägnanz sorgt. 
Auch das STERN-Bild vom Ort der Entführung in Köln war bereits auf ein Darstellungsschema bezogen worden, das im diskursiven Zusammenhang von "Terrorismus" relevant ist: Es stellt einen Tatort dar, indem es eine Reihe von Dingen in einer bestimmten Konstellation zeigt. Dabei war schon angedeutet worden, dass es sich nicht einfach nur um eine dem Schema entsprechende Tatortdarstellung handelt, sondern dass - mehr noch als bei den Bomben des SPIEGEL-Titels - die konkrete bildliche Form einen pragmatischen Mehrwert bietet. Das Bild macht durch die Positionierung der Gegenstände im Bildfeld zugleich das ,Attentat' in seinem Verlauf nachvollziehbar. Das Bild, so könnte man nun sagen, ,übererfülltt damit das Darstellungsschema und wird im Zusammenhang ikonisch prägnant. Diese ikonische Prägnanz wird im Vergleich mit einem weiteren Bild deutlich, das denselben Tatort darstellt (Abb. 11). Es handelt sich um eine Fotografie, die am Tag nach dem Attentat auf der Titelseite der BILD-Zeitung abgebildet war.

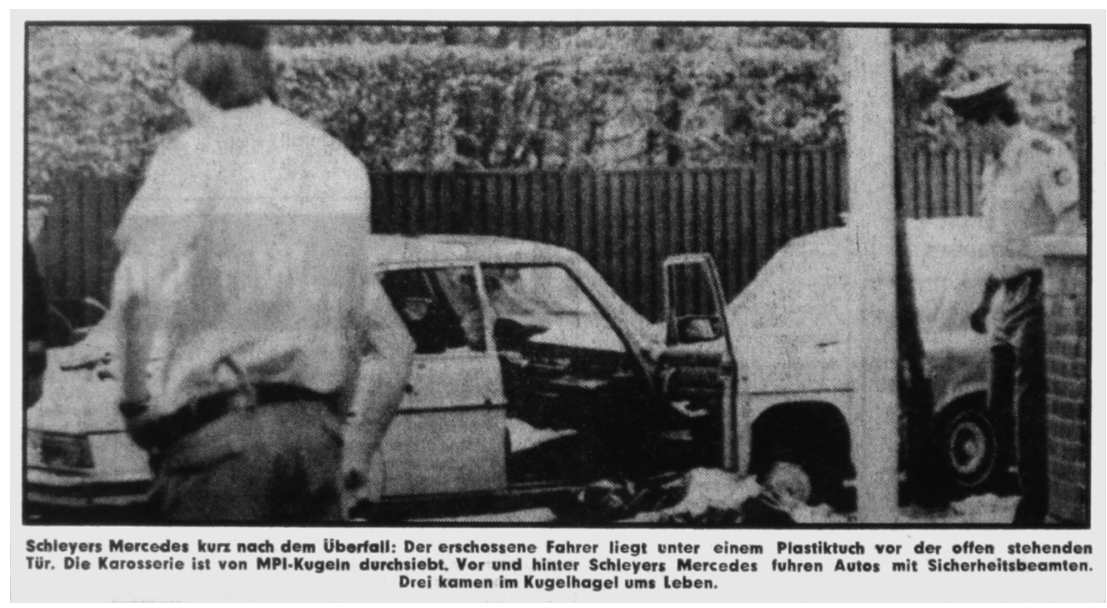

Abb. 11: BILD-Zeitung (Druck in Frankfurt), 6.9.1977, S. 1 (Ausschnitt)

Auch bei dieser Fotografie sind Autos, Leiche und Polizisten so zusammengestellt, dass das Bild gemäß dem Tatort-Schema interpretiert werden kann. Doch wird in der dargebotenen Seitenansicht auf zwei der Fahrzeuge von der Beifahrerseite der Tatverlauf gerade nicht nachvollziehbar, der auch hier in der Bildunterschrift angedeutet wird. Beim STERN-Foto ließ sich die Kreuzung von vertikalen und horizontalen Linien im Bild auf den Moment der Entführung Schleyers beziehen, bei dem seine Wagenkolonne gestoppt und seine Begleiter erschossen wurden. Beim Foto aus der BILDZeitung ist etwa die dominante weiße Linie, die das Bild im rechten Fünftel vertikal durchzieht, nicht in vergleichbarer Weise motivierbar. Sie ist allenfalls als Ausschnitt aus einem Laternenpfahl im Vordergrund erkenn- und so auf der Ebene des Zeigens interpretierbar. Im Bildzusammenhang 
durchschneidet sie jedoch die relevante Blickachse des rechts zu erkennenden Polizisten, durch die die Aufmerksamkeit auf die abgedeckte Leiche am unteren Rand gelenkt wird. Der oberflächennahe Vergleich von sichtbaren Eigenschaften - von Linienzügen und ihrer Anordnung im Rahmen der Bildfläche - liefert also Hinweise auf ein im Diskurszusammenhang unterschiedliches bildliches Überzeugungspotenzial der beiden Darstellungen desselben Tatorts. Dieser bildanalytisch gewonnene Befund wird durch die Verwendungsgeschichte der Bilder gestützt. Während das Foto der BILD-Zeitung nur dieses eine Mal erschien, werden Varianten des STERN-Fotos bis heute als Bilder des Ereignisses wiederverwendet. ${ }^{29}$ Die Tatsache, dass es gerade dieses Tatortbild ist, das bis heute als diskursiver Index ${ }^{30}$ funktioniert - als Bild, das verwendet wird, um das Ereignis der Schleyer-Entführung, die Ereignisse des sogenannten „Deutschen Herbstes“ oder gleich den ganzen Terrorismus-Zusammenhang der 1970er Jahre aufzurufen -, ist ein diskursgeschichtliches Indiz für das unterschiedliche Prägnanzpotenzial, das mit der sichtbaren Form der beiden Bilder verbunden ist.

Bei Peirce heißt es an einer Stelle im Hinblick auf das ikonische Zeichen, dass „durch seine direkte Beobachtung andere Wahrheiten über sein Objekt entdeckt werden können als jene, die für seine Konstruktion hinreichend waren". ${ }^{31}$ Diese Stelle kann man im vorliegenden Zusammenhang auch so verstehen, dass von der konkreten Ausdrucksform und ihren wahrnehmbaren Eigenschaften im Zeichenzusammenhang ein pragmatischer Mehrwert ausgehen kann, der nicht auf die Zuordnung zu einem symbolischen Schema reduzierbar ist. Dieser Fall kann daher als ikonische Prägnanz bezeichnet werden. Das Bild aus dem STERN mag einfach als Darstellung eines Tatorts entstanden sein. Die konkrete bildliche Gestalt ermöglicht es aber, in ihm mehr zu sehen. Sie ermöglicht es, anhand des statischen Bildes zugleich den Geschehensverlauf nachzuvollziehen. Die Pressefotografie entspricht also nicht nur dem Tatort-Schema, sondern wird aufgrund ihrer ikonischen Qualitäten in späteren Verwendungen zum diskursiven Index, zum Ereignisbild.

29 Ein Beispiel für eine solche Verwendung wurde oben bereits erwähnt (Abb. 9). Weitere Beispiele sind die Abbildungen auf den Umschlägen des Buchs zu Heinrich Breloers TV-Dokudrama Todessspiel (Köln 1997) und zu Anne Siemens' Geschichte des Terrorismus der 1970er Jahre aus der Perspektive der Opfer (Für die RAF war er das System, für mich der Vater. München 2007), sowie die Verwendung als einzige Abbildung im Abschnitt Terrorismus von Hermann Glasers Deutsche Kultur. Ein bistorischer Überblick von 1945 bis zur Gegenwart (München/Wien 1997, S. 325).

30 Vgl. zur Begründung dieses Terminus als einer semio-pragmatisch konsistenten Alternative zur verbreiteten Bezeichnung so verwendeter Bilder als (Foto-)Ikonen Steinseifer (im Erscheinen).

31 Peirce (1986, S. 250 f.) (Über die Einheit hypothetischer und kategorischer Propositionen, MS 787, 1897). 
Betrachtet man die Analysen zu den unterschiedlichen Ausprägungen von Prägnanzeffekten unter methodologischen Gesichtspunkten, zeigt sich noch einmal die pragmatische Grundorientierung der vorgeschlagenen Annäherung an die medienspezifische Wirkungsweise von Bildern in ihrer $\mathrm{Zu}$ sammenstellung mit Texten. Denn sowohl bei der schematischen wie auch bei der ikonischen Prägnanz ist eine Bestimmung des jeweiligen Effekts nur im Verwendungszusammenhang möglich: Unter Verwendungszusammenhang ist dabei zum einen die Zusammenstellung von Bild und Text im jeweiligen Kommunikationsangebot zu verstehen, in der transkriptiv ein Darstellungssinn fixiert wird, ${ }^{32}$ und zum anderen der größere Diskurszusammenhang, in dem einer als relevant etablierten und auch sprachlich artikulierbaren Bedeutung durch ein konkretes Bild zusätzliche Überzeugungskraft verliehen wird. Die mediale Wirkungsweise von Bildern wird in dem skizzierten theoretischen Rahmen also rekonstruktiv ${ }^{33}$ fassbar, indem zwei analytische Schritte methodisch miteinander verbunden werden: Das einzelne Bild wird zunächst einmal diskursanalytisch auf den kommunikativen Zusammenhang mit seinen Darstellungsschemata und transkriptiv etablierten Bedeutungen bezogen. Auf dieser Basis kann der formanalytische Vergleich mehrerer Bilder verdeutlichen, ob sie in den Text-Bild-Zusammenstellungen schematisch prägnant wirken und in welchem Maße sie darüber hinaus über ein ikonisches Prägnanzpotenzial verfügen.

\section{Effekte diesseits der Bilder - Ausblick auf eine pragmatische Phänomenologie}

Nach diesen Überlegungen zur Analyse von Prägnanzeffekten in Text-BildZusammenstellungen soll der Blick abschließend noch einmal zurück auf die Sprache gelenkt werden. Denn man kann sich fragen: Gibt es auch bei sprachlichen Zeichen vergleichbare Effekte der wahrnehmbaren Zeichenform, die für die Verwendung konstitutiv sind, die sich aber nicht einfach semantisch fassen lassen? Der nächstliegende Bereich, in dem man diese Frage stellen kann, ist sicher die visuelle Gestalt schriftlicher Texte. Das ist ein Bereich, der zusammen mit demjenigen der Text-Bild-Zusammenstellungen in letzter Zeit ebenfalls zunehmend die Aufmerksamkeit der Textlinguistik findet. Da Textseiten wie Bilder zweidimensionale visuelle Objekte sind, können Layoutphänomene gleichsam als bildliche Seite von Texten angesehen werden. Entsprechend heißt es bei Christa Dürscheid (2008, S. 273): „Weiter kann man den Standpunkt vertreten, dass Texte allein aufgrund ihres Layouts Bildcharakter haben." Schon der unmittelbar folgende Satz

\footnotetext{
32 Siehe erneut oben Anm. 23.

33 In Hausendorfs Sinn einer „Rekonstruktion des Prozesses der Textkonstitution“ (2008, S. 323).
} 
macht allerdings deutlich, dass der Zugriff auf diesen Bildcharakter zunächst einmal ganz selbstverständlich in einem semantischen Rahmen erfolgt, und in dem die Eigenständigkeit des bildlichen Moments als Konnotation gefasst wird: „Dieses ,Textbild“ stellt eine eigene, konnotative Bedeutungsebene dar, die den Text gewissermaßen überlagert.“

Eine semantische Analyse von Layoutphänomenen ist vielen Fällen möglich und es gibt darunter auch solche, in denen deren Eigenständigkeit so schwach ausgeprägt ist, dass kaum von einer konnotativen Bedeutungsebene auszugehen ist: Etwa bei Spiegelstrichen, die zusammen mit Einrückungen und Absätzen als Gliederungssignal für eine Reihe gleich gewichteter Aspekte stehen. Sie bilden als festgelegtes typographisches Element sogar den Ausgangs- und Bezugspunkt weitergehender sprachlicher Grammatikalisierungen, worauf Kirsten Adamzik (1995) schon vor einigen Jahren unter dem Stichwort, graphischer Stil' hingewiesen hat. Ähnliches ließe sich für Überschriften und ihr Verhältnis zum jeweiligen Haupttext zeigen.

Wenn man allerdings ganze Zeitungsseiten in den Blick nimmt, dann stoßen solche eher sprachanalogen semantischen Analysen - wie sie etwa Gunter Kress und Theo van Leeuwen im Rahmen ihrer critical layout analysis (1998) vorschlagen - an ihre Grenzen. Denn auf dieser Ebene kommen neben schematisierten Bedeutungen wiederum verstärkt Wirkungen ins Spiel, die von der konkreten Anordnung ausgehen (siehe auch Spitzmüller in diesem Band) und die auch mit Hilfe des Konnotationsbegriffs kaum zu erfassen sind. Das deutet sich auch bei Stöckl, dessen Überlegungen zu einer linguistischen Analyse der typographischen Gestalt von Texten ebenfalls semantisch ansetzen, an, wenn er schreibt:

Typographie ist relativ eng an die Funktionsweise von Sprache bzw. an den Text gebunden. Typographische Ressourcen überformen und wirken zurück auf die sprachlichen - sie operieren über dem kommunikativ-pragmatischen Phänomen Text, er ist ihr Wirkungsrabmen. (2004b, S. 21; Hervorhebung durch den Autor)

Ich möchte daher im Rahmen einer pragmatischen „Semiotik der Textgestalt“ (Raible 1991), von der Wolfgang Raible bereits vor fast 20 Jahren sprach, im Zusammenhang mit Layoutfragen ebenfalls für eine Phänomenologie der Zeichenform plädieren. Eine solche phänomenologische Perspektive, wie sie auch Heiko Hausendorf und Wolfgang Kesselheim (2008, S. 18) im Rahmen ihres textlinguistischen Entwurfs einfordern, müsste ergänzend zur ,Semantik ${ }^{6}$ einzelner Layoutelemente die mediale Wirkungsweise der sichtbaren Zeichenform stärker berücksichtigen. Dabei würde ich erneut zwei Varianten von Effekten unterscheiden, die den beiden Formen der Prägnanz entsprechen.

Das Layout einer Seite kann allein darin ,effektiv' sein, dass es durch die Gestaltung und Platzierung einzelner Textelemente die sichtbaren Differenzen bereitstellt, die nötig sind, um ein Erfassen der Textstruktur möglich 
zu machen: Die bereits erwähnten Spiegelstriche dürfen trotz der mit ihnen verbundenen grammatischen Besonderheiten als Layoutelemente nicht im Textblock untergehen, sondern um als spracbliche Gliederungshinweise funktionieren zu können, müssen sie visuell abgesetzt sein. Ist diese Bedingung erfüllt, dann kann man von einem funktional angemessenen Layout sprechen, wobei die Angemessenheit sich immer einerseits an domänenspezifischen Standards der Gestaltung - oder an „typographischen Dispositiven“, wie Stöckl (2004b, S. 43) es nennt - bemisst und andererseits am Sinn des jeweiligen Textes. Im Fall der STERN-Seite (Abb. 2) erlaubt etwa allein die Größe der Schrifttypen und die relative Position eine Unterscheidung von Überschrift und Leadtext, auch wenn die Überschrift „Das Attentat in Köln“ nicht über, sondern neben diesem Text am unteren Seitenrand steht. Dadurch werden beide allerdings zugleich auch - gemäß einem weiteren Schema - als Bildunterschriften lesbar. Die Effekte des Seitenlayouts können aber auch über eine solche textfunktionale Angemessenheit hinausgehen. Im SPIEGEL-Beispiel (Abb. 1) erlauben es die gewählten Schriftgrößen, -typen, -farben und Positionen zunächst einmal ebenfalls, den einzelnen Textteilen eine funktionale Bedeutung zuzuordnen, die dem Schema der Titelseite entspricht. Zugleich ist jedoch die spezifische Anordnung der Titelzeile nicht allein qua Angemessenheit im Rahmen dieses Schemas zu verstehen. Die Differenz in Größe und Farbe zwischen dem Substantiv Bomben und der folgenden Ortsangabe in der Bundesrepublik ist zwar schematisch betrachtet eine Gewichtung, die mit der syntaktischen Struktur korrespondiert. In besonderer Weise wirksam wird sie aber erst im konkreten Seitenzusammenhang, der in diesem Fall visuell durch das hinterlegte Bild der Bomben strukturiert wird. Das gilt noch einmal besonders für die Trennung des Wortes Bundesrepublik. Diese ist nämlich weder semantisch motiviert noch wäre sie satztechnisch notwendig - in der Zeile wäre ja noch genug Platz (vgl. Abb. 12). Plausibel wirkt sie allein durch ihre Position auf der Seite, denn hier ist sie auf die Kontur einer der Bomben bezogen. Während eine durchlaufende Zeile eher die Flächigkeit der Seite betonen würde, verstärkt der Zeilenumbruch die räumliche Wirkung der ,formatsprengenden 'Bomben und damit die Bedrohung für die Bundesrepublik, die von ihnen ausgeht.

Solche Effekte der Anordnung sind aber nicht auf den Fall beschränkt, dass ein Bild als visueller Bezugspunkt dient. Ein Beispiel dafür bietet die Titelseite der BILD-Zeitung vom 13. Mai 1972 (Abb. 13), auf der unter der Schlagzeile „Bomben-Terror“ über dieselben Gewalt-Ereignisse berichtet wird wie im SPIEGEL-Heft. Interessant am Layout der Seite sind besonders die vier gerahmten Textfelder unter der Titelzeile, die - das verdeutlicht ein weiterer Rahmen - zum selben Artikelzusammenhang gehören. Die vier Textfelder sind jeweils ähnlich aufgebaut. Sie bestehen aus einem schwarz hinterlegten oberen Teil, in dem jeweils in weiß der Name einer 

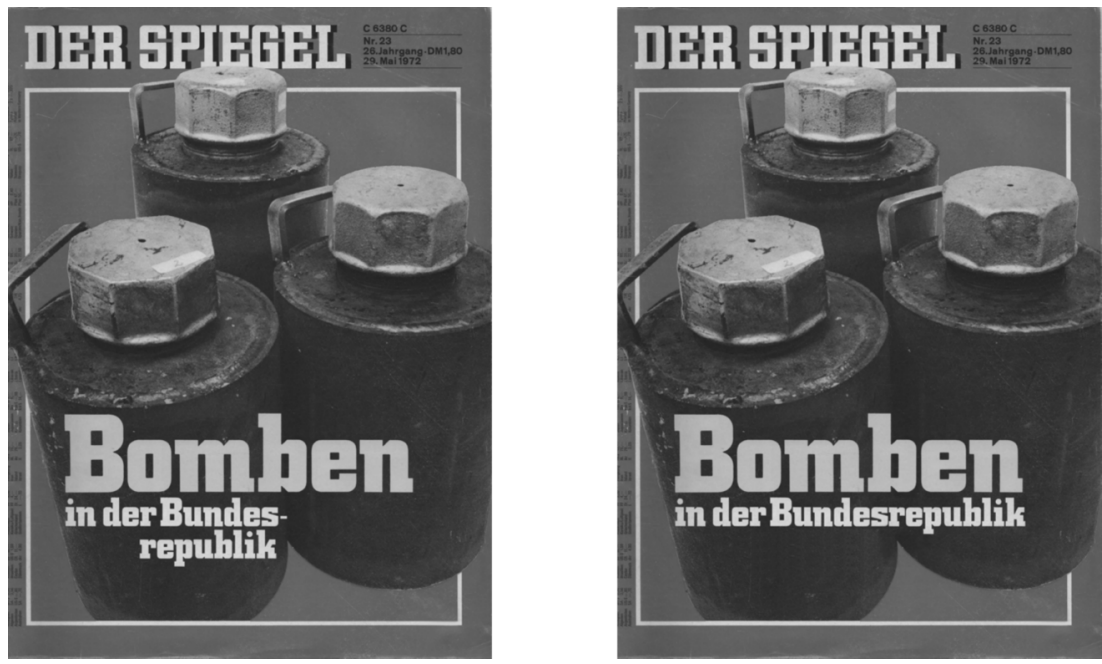

Abb. 12: SPIEGEL 23, .5.1972, Titel: links original, rechts modifiziert

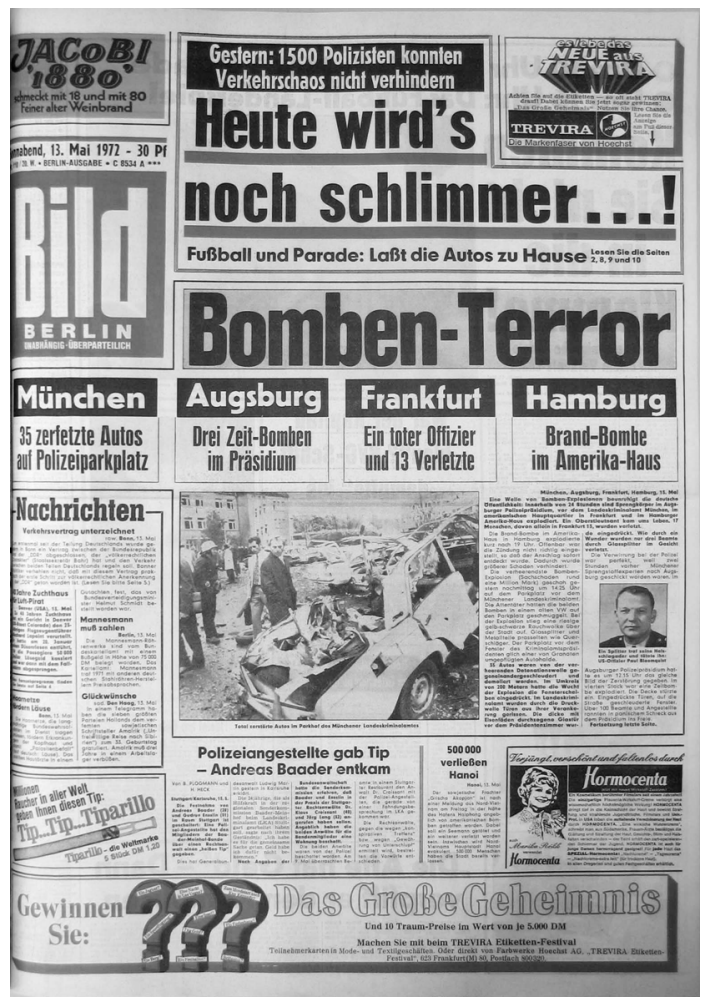

Abb. 13: BILD-Zeitung (Berlin-Ausgabe), 13.5.1972, S. 1 
Großstadt zu lesen ist und einem durch eine feine Rahmenlinie damit verbundenen unteren Teil, in dem in jeweils zwei Textzeilen auf einen Anschlag eingegangen wird, der - so ist diese Konstellation zu verstehen - in dieser Stadt stattgefunden hat. Die Felder können daher in textfunktionaler Hinsicht als visuelle Alternative zu Spiegelstrichen angesehen werden, ermöglichen sie doch wie diese die reihende Anordnung von Aspekten. Doch visuell nimmt die Reihe in Form der Kästen die ganze Breite der Seite ein sie greift nach links sogar über die Überschrift hinaus - und die Namen der Anschlagsorte sind durch Position und Farbgestaltung hervorgehoben. Durch diese Anordnung wird mit visuellen Mitteln die Gleichreitigkeit und die weite räumliche Verteilung der Anschläge deutlich gemacht und so die ubiquitäre Bedrohung durch ,Bombenterror ${ }^{6}$ - überall, jederzeit kann ein Anschlag verübt werden - im Seitenzusammenhang plausibel, auch ohne dass diese Behauptung mit sprachlichen Mitteln explizit formuliert werden muss.

Diese letzten Beispiele können nur andeuten, in welcher Weise nicht nur die kommunikative Verwendung von Bildern, sondern auch diejenige der Schrift in Texten durch die sichtbare Form der artikulierten Zeichen ,ikonisch ' mitbestimmt ist. Die vorgeschlagene phänomenologische Perspektive bietet die Möglichkeit, bei Layoutanalysen neben der Semantik einzelner Elemente die auf die Ausdrucksform bezogenen Wirkungszusammenhänge zu berücksichtigen. Gerade die Beschäftigung mit Bildern und ihren Besonderheiten kann also die pragmatische Linguistik - auf theoretischer wie methodologischer Ebene - zu einer konsequenteren Berücksichtigung der medialen Dimension von Sprache und Kommunikation hinführen. Um diesen Effekt zu erreichen, muss der Rahmen einer linguistischen Phänomenologie, der im vorliegenden Beitrag skizziert wurde, allerdings ebenso synchron und diachron durch weitere Untersuchungen an Material aus anderen Kommunikationsbereichen ausgefüllt werden, wie er im Zuge solcher Untersuchungen zu differenzieren und zu erweitern ist.

\section{Literatur}

Adamzik, Kirsten (1995): Syntax und Textgliederung. Hypotaktischer Stil, Nominalstil und graphischer Stil. In: Hindelang, Götz (Hg.): Der Gebrauch der Sprache. Festschrift für Franz Hundsnurscher zum 60. Geburtstag. Münster, S. 15-42.

Bal, Mieke (2003): Visual essentialism and the object of visual culture. In: Journal of Visual Culture 2, 1, S. 5-32.

Barthes, Roland ([1964] 1990): Rhetorik des Bildes. In: Barthes, Roland: Der entgegenkommende und der stumpfe Sinn. Kritische Essays III. Frankfurt a.M., S. $28-46$. [Zuerst in: Communications 4 (1964), S. 40-51].

Barthes, Roland ([1980] 1985): Die Helle Kammer. Bemerkung zur Photographie. Frankfurt a.M. [Zuerst: La chambre claire. Note sur la photographie. Paris 1980]. 
Belting, Hans (2001): Bild-Anthropologie - Entwürfe für eine Bildwissenschaft. München.

Boehm, Gottfried (1978): Zu einer Hermeneutik des Bildes. In: Gadamer, Hans-Georg/Boehm, Gottfried (Hg.): Seminar: Die Hermeneutik und die Wissenschaften. Frankfurt a.M., S. 444-471.

Boehm, Gottfried (1994): Die Wiederkehr der Bilder. In: Boehm, Gottfried (Hg.): Was ist ein Bild? München, S. 11-38.

Boehm, Gottfried (1999): Vom Medium zum Bild. In: Spielmann, Yvonne/Winter, Gundolf (Hg.): Bild - Medium - Kunst. München, S. 165-178.

Boehm, Gottfried (2007a): Wie Bilder Sinn erzeugen. Die Macht des Zeigens. Berlin.

Boehm, Gottfried ([2004] 2007b): Jenseits der Sprache. Anmerkungen zur Logik der Bilder. In: Boehm (2007a), S. 34-73. [Zuerst in: Maar, Christa/Burda, Hubert (Hg.): Iconic Turn. Die neue Macht der Bilder. Köln 2004, S. 28-43].

Bredekamp, Horst (2003): A neglected tradition? Art history as "Bildwissenschaft". In: Critical Inquiry 29, 3, S. 418-428.

Brink, Cornelia (1998): Ikonen der Vernichtung. Öffentlicher Gebrauch von Fotografien aus nationalsozialistischen Konzentrationslagern nach 1945. (= Schriftenreihe des Fritz-Bauer-Instituts 14). Berlin.

Cassirer, Ernst ([1929] 1954): Philosophie der symbolischen Formen. Dritter Teil: Phänomenologie der Erkenntnis. Darmstadt. [Zuerst: Berlin 1929].

Dürscheid, Christa (2008): Schrift - Text - Bild: Ein Brückenschlag. In: Zeitschrift für germanistische Linguistik 35, 3, S. 269-282.

Elkins, James (2003): Visual studies: a skeptical introduction. New York u.a.

Fehrmann, Gisela/Linz, Erika (2008): Der hypnotische Blick. Zur kommunikativen Funktion deiktischer Zeichen. In: Wenzel/Jäger (Hg.), S. 261-288.

Fix, Ulla (1996): Textstile und KonTextstile. Stil in der Kommunikation als umfassende Semiose von Sprachlichem, Parasprachlichem und Außersprachlichem. In: Fix, Ulla/ Lerchner, Gotthard (Hg.): Stil und Stilwandel. (= Leipziger Arbeiten zur Sprachund Kommunikationsgeschichte 3). Frankfurt a.M., S. 111-132.

Göpferich, Susanne (2008): Textverstehen und Textverständlichkeit. In: Janich, Nina (Hg.): Textlinguistik. 15 Einführungen. Tübingen, S. 291-312.

Hausendorf, Heiko (2008): Zwischen Linguistik und Literaturwissenschaft: Textualität revisited. Mit Illustrationen aus der Welt der Urlaubsansichtskarte. In: Zeitschrift für germanistische Linguistik 36, 3, S. 319-342.

Hausendorf, Heiko/Kesselheim, Wolfgang (2008): Textlinguistik fürs Examen. (= Linguistik fürs Examen 5). Göttingen.

Hermann, Kai/Koch, Peter (1977): Entscheidung in Mogadischu: die 50 Tage nach Schleyers Entführung. Dokumente, Bilder, Zeugen. Hamburg.

Holly, Werner (2005): Zum Zusammenspiel von Sprache und Bildern im audiovisuellen Verstehen. In: Busse, Dietrich/Niehr, Thomas/Wengeler, Martin (Hg.): Brisante Semantik. Neuere Konzepte und Forschungsergebnisse einer kulturwissenschaftlichen Semantik. (= Reihe germanistische Linguistik 259). Tübingen, S. 373-353. 
Holly, Werner (2009): Der Wort-Bild-Reißverschluss. Über die performative Dynamik der audiovisuellen Transkriptivität. In: Feilke, Helmuth/Linke, Angelika (Hg.): Oberfläche und Performanz. (= Reihe germanistische Linguistik 283). Tübingen, S. 93-110.

Hüppe, Angelika (1984): Prägnanz - Ein gestalttheoretischer Grundbegriff. Experimentelle Untersuchungen. München.

Husserl, Edmund ([1913] 1992): Ideen zu einer reinen Phänomenologie. (= Gesammelte Schriften 5). Hamburg. [Zuerst: Halle 1913].

Imdahl, Max ([1980] 1996): Kontingenz - Komposition - Providenz. Zur Anschauung eines Bildes von Giotto. In: Imdahl, Max: Gesammelte Schriften. Bd. 3: Reflexion - Theorie - Methode. Hrsg. v. Gottfried Boehm. Frankfurt a.M., S. 464-500. [Zuerst in: neue hefte für philosophie 18-19 (1980), S. 151-177].

Jäger, Ludwig (2002): Transkriptivität. Zur medialen Logik der kulturellen Semantik. In: Jäger, Ludwig/Stanitzek, Georg (Hg.): Transkribieren - Medien/Lektüre. München, S. 19-41.

Jäger, Ludwig (2008): Indexikalität und Evidenz. Skizze zum Verhältnis von referentieller und inferentieller Bezugnahme. In: Wenzel/Jäger (Hg.), S. 289-315.

Kemp, Wolfgang ([1985] 1996): Kunstwerk und Betrachter: Der rezeptionsästhetische Ansatz. In: Belting, Hans et al. (Hg.): Kunstgeschichte. Eine Einführung. 5., überarb. Aufl. Berlin, S. 240-257. [1. Aufl. Berlin 1985].

Koetzle, Hans-Michael (2002): Photo Icons. Die Geschichte hinter den Bildern. 1827 1991. 2 Bde. Köln.

Kraushaar, Wolfgang (Hg.) (2006): Die RAF und der Linke Terrorismus. 2 Bde. Hamburg.

Kress, Gunther/van Leeuwen, Theo (1996): Reading images. The grammar of visual design. London/New York.

Kress, Gunther/van Leeuwen, Theo (1998): Front pages: (The critical) analysis of newspaper layout. In: Bell, Alan/Garrett, Peter (Hg.): Approaches to media discourse. Oxford, S. 186-219.

Linke, Angelika/Nussbaumer, Markus/Portmann, Paul R. (2004): Studienbuch Linguistik. 5., erw. Aufl. (= Reihe germanistische Linguistik 121). Tübingen.

Lohmar, Dieter (1993): Grundzüge eines Synthesis-Modells der Auffassung: Kant und Husserl über den Ordnungsgrad sinnlicher Vorgegebenheiten und die Elemente einer Phänomenologie der Auffassung. In: Husserl-Studies 10, S. 111-141.

Mitchell, W.J. Thomas (1994): Picture theory. Essays on verbal and visual representation. Chicago/London.

Muckenhaupt, Manfred (1986): Text und Bild: Grundfragen der Beschreibung von TextBild-Kommunikationen. (= Tübinger Beiträge zur Linguistik 271). Tübingen.

Nöth, Winfried (2000): Handbuch der Semiotik. 2., vollst. neu bearb. u. erw. Aufl. mit 89 Abbildungen. Stuttgart/Weimar.

Peirce, Charles Sanders (1983): Phänomen und Logik der Zeichen. Herausgegeben und übersetzt von Helmut Pape. Frankfurt a.M. 
Peirce, Charles Sanders (1986): Semiotische Schriften. Bd. 1. Frankfurt a.M.

Raible, Wolfgang (1991): Die Semiotik der Textgestalt. Erscheinungsformen und Folgen eines kulturellen Evolutionsprozesses. Heidelberg.

Sachs-Hombach, Klaus (2003): Das Bild als kommunikatives Medium. Elemente einer allgemeinen Bildwissenschaft. Köln.

Schmidt, Siegfried J. ([1980] 1991): Grundriß der Empirischen Literaturwissenschaft. Frankfurt a.M. [Zuerst: Braunschweig 1980].

Schmitz, Ulrich (2003): Lesebilder im Internet. Neue Koalitionen und Metamorphosen zwischen Text und Bild. In: Zeitschrift für Germanistik. Neue Folge 3, S. $605-628$.

Schmitz, Ulrich (2005): Blind für Bilder. Warum sogar Sprachwissenschaftler auch Bilder betrachten müssen. In: Osnabrücker Beiträge zur Sprachtheorie 69, S.187-227.

Schmitz, Ulrich (2008): Bildakte? How to do Things with Pictures. In: Zeitschrift für Germanistische Linguistik 35, 3, S. 419-433.

Schmitz, Ulrich/Wenzel, Horst (Hg.) (2003): Wissen und neue Medien. Bilder und Zeichen von 800 bis 2000. (= Philologische Studien und Quellen 177). Berlin.

Sonesson, Göran (1993): Die Semiotik des Bildes: Zum Forschungsstand am Anfang der 90er Jahre. In: Zeitschrift für Semiotik 15/1-2, S. 127-160.

Spitzmüller, Jürgen (i.d.Bd.): Typografische Variation und (Inter-)Medialität. Zur kommunikativen Relevanz skripturaler Sichtbarkeit.

Steinseifer, Martin (2009): Sichtbar verkörperte Artikulationen - Was wird aus dem Text(-Begriff) angesichts der Konjunktur des Performativen? In: Buss, Mareike et al. (Hg.): Theatralität des sprachlichen Handelns. Eine Metaphorik zwischen Linguistik und Kulturwissenschaften. München, S. 143-163.

Steinseifer, Martin (i.Ersch.): Ereignisbilder - Zum Verhältnis von Indexikalität, Symbolizität und Ikonizität bei Pressefotografien. In: Boehm, Gottfried/Egenhofer, Sebastian/Spies, Christian (Hg.): Zeigen. Die Rhetorik des Sichtbaren. München.

Stöckl, Hartmut (2004a): Die Sprache im Bild - Das Bild in der Sprache. Zur Verknüpfung von Sprache und Bild im massenmedialen Text. (= Linguistik - Impulse \& Tendenzen 3). Berlin/New York.

Stöckl, Hartmut (2004b): Typographie: Gewand und Körper des Textes - Linguistische Überlegungen zu typographischer Gestaltung. In: Zeitschrift für Angewandte Linguistik 41, S. 5-48.

Wenzel, Horst/Jäger, Ludwig (Hg.) (2008): Deixis und Evidenz. (= Rombach Wissenschaften, Reihe Scenae 8). Freiburg i.Br.

Wiesing, Lambert (2005): Artifizielle Präsenz. Studien zur Philosophie des Bildes. Frankfurt a.M. 